ALEA, Lat. Am. J. Probab. Math. Stat. 18, 727-760 (2021)

DOI: $10.30757 /$ ALEA.v18-27

\title{
Hitting probabilities for Lévy processes on the real line
}

\section{Tomasz Grzywny, Łukasz Leżaj and Maciej Miśta}

Wrocław University of Science and Technology

Faculty of Pure and Applied Mathematics

Wyb. Wyspiańskiego 27

50-370 Wrocław, Poland

E-mail address: tomasz.grzywny@pwr.edu.pl, lukasz.lezaj@pwr.edu.pl,

maciej.mista@pwr.edu.pl

Abstract. We prove sharp two-sided estimates on the tail probability of the first hitting time of bounded interval as well as its asymptotic behaviour for general non-symmetric processes which satisfy an integral condition

$$
\int_{0}^{\infty} \frac{d \xi}{1+\operatorname{Re} \psi(\xi)}<\infty
$$

To this end, we first prove and then apply the global scale invariant Harnack inequality. Results are obtained under certain conditions on the characteristic exponent. We provide a wide class of Lévy processes which satisfy these assumptions.

\section{Introduction}

The aim of this paper is to discuss the distribution of the first hitting time of the point or the bounded interval for non-symmetric Lévy processes which satisfy the following condition:

$$
\int_{0}^{\infty} \frac{d \xi}{1+\operatorname{Re} \psi(\xi)}<\infty
$$

Such condition implies that 0 is regular for itself. Under some regularity assumptions we prove sharp two-sided estimates on the tail probability of the first hitting time of a point or a bounded interval, as well as its asymptotic behaviour. In the case of symmetric processes, it can be described by the compensated potential

Received by the editors May 8th, 2020; accepted January 5th, 2021.

2010 Mathematics Subject Classification. 60G51, 60J45, 60J50, 60J75.

Key words and phrases. Lévy process, Lévy-Khintchine exponent, hitting time, Harnack inequality, Green function, renewal function.

The research was partially supported by National Science Centre (Poland): grant 2015/17/B/ST1/01043. 
kernel (see Grzywny and Ryznar, 2017), which is given by

$$
K(x)=\int_{0}^{\infty}(p(s, 0)-p(s, x)) d s,
$$

where $p(s, \cdot)$ is the transition density of the process (which exists due to the integral condition). However, in our setting one of substantial difficulties one has to overcome is the fact that we do not a priori know if $K$ exists, and even if it does exist, it may vanish on the whole half-line, an example being a one-dimensional completely asymmetric point recurrent stable process, i.e. with the stability index $\alpha \in(1,2)$ and skewness parameter $\beta= \pm 1$ (see the formula for the compensated potential kernel in Port (1967, page 372)). For symmetric Lévy processes its existence is an easy consequence of the monotone convergence theorem, but in our case we are, in general, forced to adopt a different method. Instead, we propose an approach based on the symmetrized compensated potential kernel

$$
H(x)=\frac{1}{\pi} \int_{0}^{\infty}(1-\cos x s) \operatorname{Re}\left[\frac{1}{\psi(s)}\right] d s,
$$

which turns out to be the proper object for description of the behaviour of the first hitting time. Its huge advantage is the fact that the integrability condition we assume in the whole paper ensures that $H$ is well-defined and therefore can serve our purpose.

Let us briefly describe our results. First, we concentrate on the asymptotic behaviour of the first hitting time of arbitrary compact sets which contain the origin (Theorem 4.7 and Corollary 4.8). The obtained asymptotics hold true if $\operatorname{Re} \psi$ varies regularly with parameter $\alpha \in(1,2]$ and $\operatorname{Im} \psi$ displays a similar behaviour. We devote Section 3 to discussion about situations in which such condition holds true (see Theorem 3.4). In particular, that turns out to be true if the Lévy measure is of the special form

$$
\nu(d x)=C_{d} \mathbf{1}_{x<0} \nu_{0}(d x)+C_{u} \mathbf{1}_{x>0} \nu_{0}(d x),
$$

where $\nu_{0}(d x)$ is a symmetric Lévy measure. An important class of processes which clearly exhibit such behaviour are spectrally one-sided Lévy processes; in such case we simply have either $C_{d}=0$ (spectrally positive case) or $C_{u}=0$ (spectrally negative case). For the sake of completeness we also note that $C_{u}=C_{d}$ gives rise to a symmetric process.

Next, we turn our attention to derivation of sharp two-sided estimates on the first hitting time. To that end, we prove the global scale invariant Harnack inequality under weak lower scaling condition on the real part of the characteristic exponent. That result seems to be of some value in and of itself, as Harnack inequality is very strong theoretic tool in the potential theory. For instance, its easy consequences are estimates on the derivative of the renewal function for the ladder height process, which in turn entail estimates on the density of the distribution of the supremum process (see Chaumont and Małecki, 2016). We also provide estimates on the boundary behaviour of harmonic functions. Meanwhile, we derive estimates on the Green function of a bounded interval and a half-line (Lemma 5.1 and Corollary 5.6), which also seem to be interesting on its own. For example, the first one together with some regularity assumptions on the Lévy measure imply the boundary Harnack inequality, which is also an important potential theoretic tool (see e.g. Bogdan et al., 2015). 
Eventually, we apply those results to obtain estimates on the tail of the first hitting time of points and intervals. They are derived under assumptions of global lower scaling property, zero mean and control of $K^{\lambda}$ from below by $H$ for small $\lambda$ (see (2.8) for definition). The estimates are expressed in terms of symmetrized compensated potential kernel $H$ and renewal function for the dual process $\widehat{V}$, and therefore, in particular, do not require the existence of the compensated potential kernel $K$. Nevertheless, in Section 4 we provide conditions which assure that $K$ is well-defined. We remark here that if the process is symmetric, then our assumptions reduce to those obtained in Grzywny and Ryznar (2017). Since the third assumption is not a priori obvious for general non-symmetric processes, in Subsection 6.1 we present an example of wide class of processes for which such property holds true. Furthermore, if we restrict ourselves to the special case of spectrally negative Lévy processes then due to its specific structure, we are able to prove sharp two-sided estimates on both sides of the interval and for any $t>0$ (see Corollary 6.13). To our best knowledge, such results have not been obtained before. Apart from its own value, they can be used, together with heat kernel estimates (Grzywny and Szczypkowski, 2020) for instance for estimation of the Hausdorff dimension of the inverse images of Lévy processes (see Park et al., 2020). We also remark that although our main object to operate with is the real part of the characteristic exponent, one can work with the tail of the Lévy measure instead, since in view of Grzywny et al. (2018, Proposition 3.8), scaling property of the latter implies scaling of the former.

The first studies on the first hitting time of a point or a compact set concerned $\alpha$-stable processes. The asymptotic behaviour in the case of recurrent $\alpha$-stable process, i.e. $1<\alpha \leqslant 2$, for arbitrary compact sets, was derived in Port (1967). Next, in Yano et al. (2009) the authors discuss the law of the first hitting time of a point for the symmetric $\alpha$-stable processes with $1<\alpha \leqslant 2$. A series representation of the density of the first hitting time of a point in the case of spectrally positive $\alpha$ stable Lévy processes, $1<\alpha<2$, was obtained in Peskir (2008) and Simon (2011). That result was extended two general spectrally two-sided $\alpha$-stable processes with $1<\alpha<2$ in Kuznetsov et al. (2014). Let us note here that in case of spectrally negative Lévy processes starting from the left side of the interval, the first hitting time is equal to the first passage time through the left end and in consequence, one may apply tools from the fluctuation theory to handle the problem (see e.g. Bertoin (1996) or Sato (1999) for details).

The general symmetric case is much harder to handle and in principle, requires some regularity assumptions on the characteristic exponent of the process. In Kwaśnicki (2012) the author derives an integral representation of the distribution function of the first hitting time of a point in terms of eigenfunctions of the semigroup of the process killed upon hitting the origin. That idea was later adopted in Juszczyszyn and Kwaśnicki (2015) to obtain the asymptotic expansion of the distribution function (and its derivatives) of the first hitting time of a point for symmetric Lévy processes with completely monotone jumps. Recently, in Mucha (2019), the ideas from Kwaśnicki (2012) were extended to non-symmetric Lévy processes. A different approach was proposed in Grzywny and Ryznar (2017), where the authors prove and apply the global Harnack inequality in order to obtain sharp estimates on the tail probability of the first hitting time of points and bounded intervals for symmetric processes under global lower scaling condition imposed on 
the characteristic exponent. In the present paper we generalize these ideas to nonsymmetric Lévy processes, which, to our best knowledge, has not been investigated in such generality before.

The article is organized as follows. In Section 2 we introduce our setting, basic objects and tools exploited in the paper and prove some auxiliary results. In Section 3 we prove that some specific form of the Lévy measure and regular variation of $\operatorname{Re} \psi$ implies that $\operatorname{Im} \psi$ is in fact, comparable to the real part; we apply that result in Section 4, where asymptotic behaviour of the tail of the distribution of the first hitting time is obtained. Here, Theorem 3.4 provides an important example. Section 5 is devoted to the proof of the Harnack inequality, some of its consequences and boundary behaviour of harmonic functions. We apply those results in Section 6 , where we provide sharp two-sided estimates on the tail probability of the first hitting time of a bounded interval. Finally, in Subsection 6.1 we indicate a wide class of non-symmetric processes which satisfy assumptions of Theorem 6.10.

\section{Preliminaries}

Notation. Throughout the paper $c, c_{1}, C_{1}, \ldots$ denote positive constants which may vary from line to line during estimates. We write $c=c(a)$ when $c$ depends only on parameter $a$. For two numbers $a, b$ we denote $a \wedge b=\min \{a, b\}$. For two functions $f, g$ we write $f \approx g$ if the ratio $f(u) / g(u)$ stays between two positive constants. That notation gives rise to the notion of sharp estimates. Namely, we say that a sharp two-sided estimate on a function $f$ holds if there is a function $g$ and a constant $c>0$ such that

$$
c^{-1} g(x) \leqslant f(x) \leqslant c g(x)
$$

or, in terms of the definition above, $f \approx g$. Similarly, we write $f \lesssim g(f \gtrsim g)$ if the ratio is bounded from above (below) by a positive constant. By analogy, this corresponds to one-sided (upper or lower) estimates. By $f \cong c g, x \rightarrow x_{0}$, we mean that $\lim _{x \rightarrow x_{0}} f(x) / g(x)=c$. For positive $g$ we write $f(x)=\mathcal{O}(g(x))$, $x \rightarrow x_{0}$, if $\limsup _{x \rightarrow x_{0}}|f(x)| / g(x)<\infty$. For a complex-valued function $f: \mathbb{R} \mapsto \mathbb{C}$, by $f^{-1}$ we denote its generalized inverse, that is $f^{-1}(s)=\sup \left\{r>0: f^{*}(r)=s\right\}$, where $f^{*}(r)=\sup _{|x| \leqslant r} \operatorname{Re} f(x)$. Borel sets on the real line are denoted by $\mathcal{B}_{\mathbb{R}}$. Finally, for $r>0$ by $B_{r}$ we denote a ball centered at the origin and of radius $r$, i.e. $B_{r}=\{x \in \mathbb{R}:|x|<r\}$.

Throughout the whole paper we let $\mathbf{X}=\left(X_{t}: t \geqslant 0\right)$ be a one-dimensional Lévy process with the Lévy measure $\nu$. Recall that any Lévy measure satisfies the following conditions:

$$
\nu(\{0\})=0 \quad \text { and } \quad \int_{\mathbb{R}}\left(1 \wedge x^{2}\right) \nu(d x)<\infty .
$$

If we let $\psi$ be its characteristic exponent, that is

$$
\mathbb{E} e^{i \xi X_{t}}=e^{-t \psi(\xi)}, \quad \xi \in \mathbb{R},
$$

then it is well known that $\psi$ is of the form

$$
\psi(\xi)=\sigma^{2} \xi^{2}-i \gamma \xi-\int_{\mathbb{R}}\left(e^{i \xi z}-1-i \xi z \mathbf{1}_{|z|<1}\right) \nu(d z), \quad \xi \in \mathbb{R},
$$

where $\sigma \geqslant 0$ and $\gamma \in \mathbb{R}$. Note that since we do not assume symmetry of $X_{t}$, both $\nu$ and $\psi$ need not be symmetric. The triplet $(\sigma, \gamma, \nu)$ uniquely determines the Lévy 
process and is therefore called the generating triplet of $\mathbf{X}$. Let us notice that

$$
\operatorname{Re} \psi(\xi)=\sigma^{2} \xi^{2}+\int_{\mathbb{R}}(1-\cos \xi z) \nu(d z), \quad \xi \in \mathbb{R},
$$

and

$$
\operatorname{Im} \psi(\xi)=-\gamma \xi+\int_{\mathbb{R}}\left(\xi z \mathbf{1}_{|z|<1}-\sin \xi z\right) \nu(d z), \quad \xi \in \mathbb{R} .
$$

Observe that $\operatorname{Re} \psi$ is symmetric even if $\mathbf{X}$ is not symmetric. If we assume that $\int_{(-1,1)^{c}}|z| \nu(d z)<\infty$, then we can also write

$$
\operatorname{Im} \psi(\xi)=-\gamma_{1} \xi+\int_{\mathbb{R}}(\xi z-\sin \xi z) \nu(d z),
$$

where $\gamma_{1}=\gamma+\int_{(-1,1)^{c}} z \nu(d z)$. In particular, if $\mathbb{E} X_{1}=0$ then $\gamma_{1}=0$.

Our standing assumption is finiteness of the following integral:

$$
\int_{0}^{\infty} \frac{d \xi}{1+\operatorname{Re} \psi(\xi)}<\infty
$$

Such condition implies that $\operatorname{Re} \psi$ is unbounded, hence it must not be a characteristic exponent of compound Poisson process. It follows that $\operatorname{Re} \psi(\xi)>0$ for $\xi \neq 0$, since otherwise we would have $\sigma=0$ in (2.2) and $\nu(d x)$ would be supported on $\left\{\frac{2 k \pi}{\xi}: k \in\right.$ $\mathbb{Z} \backslash\{0\}\}$ for some $\xi \neq 0$. In particular, due to Lévy condition (2.1) we would conclude that $\nu(\mathbb{R})<\infty$, which is the case for compound Poisson process only. Furthermore, since $e^{-x} \leqslant(1+x)^{-1}$ for $x \geqslant 0$, we obtain that $\left|e^{-t \psi(\cdot)}\right|=e^{-t \operatorname{Re} \psi(\cdot)}$ is integrable. Thus, by the Fourier inversion formula, the transition density $p(t, \cdot)$ of $X_{t}$ exists for all $t>0$ and is given by

$$
p(t, x)=\frac{1}{2 \pi} \int_{\mathbb{R}} \operatorname{Re}\left[e^{-t \psi(\xi)-i \xi x}\right] d \xi, \quad x \in \mathbb{R} .
$$

If, following Pruitt (1981), we define the concentration function by setting for $r>0$,

$$
h(r)=\frac{\sigma^{2}}{r^{2}}+\int_{\mathbb{R}}\left(1 \wedge \frac{|s|^{2}}{r^{2}}\right) \nu(d s)
$$

then by Grzywny (2014, Lemma 4), for all $x \in \mathbb{R}$,

$$
h(1 /|x|) \approx \psi^{*}(|x|)
$$

Recall that $h$ is continuous and strictly decreasing. Lastly, also due te Pruitt we introduce the compensated drift part by setting

$$
b_{r}=\gamma+\int_{\mathbb{R}} z\left(\mathbf{1}_{|z|<r}-\mathbf{1}_{|z|<1}\right) \nu(d z) .
$$

We now turn to introduction of basic objects from the potential theory. For any $x \in \mathbb{R}, \mathbb{P}^{x}$ and $\mathbb{E}^{x}$ will denote the distribution and the expectation for the process $\mathbb{X}+x$, with the standard notation $\mathbb{P}^{0}=\mathbb{P}$ and $\mathbb{E}^{0}=\mathbb{E}$. We also write $\mathbb{E}^{x}[A ; Y]=$ $\mathbb{E}^{x} \mathbf{1}_{A} Y$. By $\tau_{D}$ we denote the first exit time from an open set $D$, i.e.

$$
\tau_{D}=\inf \left\{t>0: X_{t} \notin D\right\} .
$$

For a closed set $F$ we define the first hitting time of $F$ as the first exit time from its complement $F^{c}$, that is $T_{F}=\tau_{F^{c}}$. If $F=\{b\}$ is a singleton, then slightly abusing 
the notation, we write $T_{b}=T_{\{b\}}$. For $\lambda>0$ we let $u^{\lambda}$ be the $\lambda$-potential kernel, that is the Laplace transform of $p(\cdot, x)$ :

$$
u^{\lambda}(x)=\int_{0}^{\infty} e^{-\lambda t} p(t, x) d t .
$$

If $u^{\lambda}$ exists for $\lambda=0$ then we set $u^{0}=u$ and then the process is transient (see Bertoin (1996, Theorem I.17)). In general, this needs not be the case (take for example the classical stable process with stability index $\alpha>1$, which is recurrent). Nevertheless, the condition

$$
\int_{0}^{\infty} \frac{d \xi}{1+\operatorname{Re} \psi(\xi)}<\infty
$$

together with Bretagnolle (1971, Theoreme 7 and 8), implies that 0 is regular for itself, that is

$$
\mathbb{P}^{0}\left(T_{0}=0\right)=1 .
$$

That in turn, combined with Kesten (1969, Theorem 1$)$, yield that $\mathbb{P}^{x}\left(T_{0}<\infty\right)>0$ for any $x \in \mathbb{R}$.

Let us also set

$$
\kappa=\lim _{\lambda \rightarrow 0^{+}} \frac{1}{u^{\lambda}(0)}=\lim _{\lambda \rightarrow 0^{+}} \frac{1}{2 \pi}\left(\int_{\mathbb{R}} \operatorname{Re}\left[\frac{1}{\lambda+\psi(\xi)}\right] d \xi\right)^{-1} .
$$

Clearly, $\kappa \in[0, \infty)$. Moreover, from Bertoin (1996, Theorem I.17) it follows that the process is transient if $\kappa>0$.

By Bertoin (1996, Corollary II.18 and Theorem II.19) we get that $x \mapsto \mathbb{E}^{x} e^{-\lambda T_{0}}$ is continuous with respect to $x$, and for any $x \in \mathbb{R}$ we have

$$
u^{\lambda}(x)=u^{\lambda}(0) \mathbb{E}^{-x} e^{-\lambda T_{0}} .
$$

Note that for recurrent processes we have $u(x)=\infty$ for all $x \in \mathbb{R}$. Instead one can define the compensated $\lambda$-potential kernel by setting for $\lambda>0$

$$
K^{\lambda}(x)=u^{\lambda}(0)-u^{\lambda}(x), \quad x \in \mathbb{R} .
$$

In view of (2.7) we get that $K^{\lambda} \geqslant 0$ for all $\lambda \geqslant 0$.

The next natural move would be to pass with $\lambda$ to 0 and define the compensated potential kernel

$$
K(x)=\lim _{\lambda \rightarrow 0^{+}} K^{\lambda}(x), \quad x \in \mathbb{R} .
$$

In general, however, it is not easy to show even the existence of $K$, let alone its further properties. For some elaboration on that subject, including special cases when $K$ can be well-defined, see Section 4. If it does exist then one can express the probability of not hitting the origin in terms of $K$ and $\kappa$.

Proposition 2.1. Suppose that $K$ exists. Then

$$
\mathbb{P}^{x}\left(T_{0}=\infty\right)=\kappa K(-x) .
$$

If $\kappa=0$ then $\mathbb{P}^{x}\left(T_{0}<\infty\right)=1$ for all $x \in \mathbb{R}$.

It follows that if $K$ exists and $\mathbb{P}^{x}\left(T_{0}<\infty\right)=1$ then $\mathbf{X}$ is recurrent. 
Proof: Observe that

$$
\lim _{\lambda \rightarrow 0^{+}} \mathbb{E}^{x} e^{-\lambda T_{0}}=\mathbb{P}^{x}\left(T_{0}<\infty\right)
$$

On the other hand,

$$
\mathbb{E}^{x} e^{-\lambda T_{0}}=\frac{u^{\lambda}(-x)}{u^{\lambda}(0)}=1-\frac{u^{\lambda}(0)-u^{\lambda}(-x)}{u^{\lambda}(0)} \rightarrow 1-\kappa K(-x),
$$

as $\lambda \rightarrow 0^{+}$.

Instead of $K$, let us consider the symmetrized $\lambda$-potential kernel

$$
H^{\lambda}(x)=K^{\lambda}(x)+K^{\lambda}(-x)
$$

By Bertoin (1996, Theorem II.19),

$$
H^{\lambda}(x)=\frac{1}{\pi} \int_{\mathbb{R}}(1-\cos x s) \operatorname{Re}\left[\frac{1}{\lambda+\psi(s)}\right] d s .
$$

Under (2.4) one can show that the symmetrized compensated potential kernel

$$
H(x)=\lim _{\lambda \rightarrow 0^{+}} H^{\lambda}(x), \quad x \in \mathbb{R}
$$

is well-defined and

$$
H(x)=\frac{1}{\pi} \int_{\mathbb{R}}(1-\cos x s) \operatorname{Re}\left[\frac{1}{\psi(s)}\right] d s, \quad x \in \mathbb{R} .
$$

Indeed, it follows immediately from the fact that $\operatorname{Re} \psi(\xi) \gtrsim \xi^{2}$ for $|\xi| \leqslant 1$ (see e.g. Sato, 1999, proof of Theorem 37.8), which together with (2.4) justify the application of the dominated convergence theorem and the claim follows. Proceeding as in the proof of Grzywny and Ryznar (2017, Proposition 2.2) one may prove that $H$ is subadditive on $\mathbb{R}$. See also Pantí (2017, Proposition 3.7) for a similar result, although derived under different assumptions.

We will often assume the so-called global weak lower scaling condition on the real part of the characteristic exponent with the scaling index strictly bigger than 1. We will say that a function $f$ satisfies the global weak lower scaling condition, if there are $\alpha \in \mathbb{R}$ and $c \in(0,1]$ such that for all $u>0$ and $\lambda \geqslant 1$,

$$
f(\lambda u) \geqslant c \lambda^{\alpha} f(u), \quad u>0 .
$$

We will write shortly $f \in \operatorname{WLSC}(\alpha, c)$. The pair $(\alpha, c)$ will be referred to as scaling characteristics or simply scalings. Note that by Bogdan et al. (2014, Lemma 11), $f \in \operatorname{WLSC}(\alpha, c)$ for some $\alpha \in \mathbb{R}$ and $c \in(0,1]$ if and only if the function

$$
(0, \infty) \ni x \mapsto x^{-\alpha} f(x)
$$

is almost increasing. See e.g. Bogdan et al. (2014, Section 3) for details. Clearly, the condition $\operatorname{Re} \psi \in \operatorname{WLSC}(\alpha, c)$ for some $\alpha>1$ and $c \in(0,1]$, implies (2.4). Moreover, by (2.5) and Grzywny and Szczypkowski (2020, Theorem 3.1), for all $x \in \mathbb{R}$,

$$
\operatorname{Re} \psi(x) \approx \psi^{*}(|x|) \approx h(1 /|x|) .
$$

We note that under assumptions of the global scaling property of the real part of the characteristic exponent, its control over the imaginary part (see Grzywny, 2019, Lemma 12) and vanishing of the first moment, i.e. $\mathbb{E} X_{1}=0$, one can show that $\int_{0}^{1} \operatorname{Re}(1 / \psi(\xi)) d \xi=\infty$ and, in view of Bertoin (1996, Theorem I.17), conclude that $\mathbf{X}$ is recurrent, and consequently, $u(x)=\infty$ for all $x \in \mathbb{R}$. As the processes 
we study in this paper often satisfy such assumptions, we are in dire need of some object alternative to the (infinite) potential kernel. The symmetrized compensated potential kernel $H$ can be of usage here, but it appears that the (ordinary) compensated kernel $K$ is more appropriate for description of hitting probability behaviour. The only problem is that in general we are not able to determine whether it exists. We devote Sections 4 and 6 to the detailed discussion on the subject.

We note one simple yet crucial observation.

Proposition 2.2. Suppose that $\mathbb{E} X_{1}=0$ and $\operatorname{Re} \psi \in \operatorname{WLSC}(\alpha, \chi)$ for some $\alpha>1$ and $\chi \in(0,1]$. There is $c \geqslant 1$ such that for all $r>0$,

$$
c^{-1} \frac{1}{r h(r)} \leqslant H(r) \leqslant c \frac{1}{r h(r)} .
$$

In particular, $H \in \mathrm{WLSC}(\alpha-1, \tilde{\chi})$ for some $\tilde{\chi} \in(0,1]$.

Proof: Observe that by Grzywny (2019, Lemma 12), for any $r>0$,

$$
H(r) \approx \int_{0}^{\infty}(1-\cos r s) \frac{1}{\operatorname{Re} \psi(s)} d s .
$$

Now, the claim follows by Grzywny (2019, Lemma 13).

Apart from free processes, for an open set $D$ one can consider the process killed when exiting $D$, denoted by $X_{t}^{D}$. Namely, for any measurable function $f$,

$$
\mathbb{E}^{x} f\left(X_{t}^{D}\right)=\mathbb{E}^{x}\left[t<\tau_{D} ; f\left(X_{t}\right)\right], \quad t>0, x \in \mathbb{R} .
$$

By analogy, by $p^{D}(t ; \cdot, \cdot)$ we denote its transition density. It is then known that

$$
p^{D}(t ; x, y)=p(t, y-x)-\mathbb{E}^{x}\left[t>\tau_{D} ; p\left(t-\tau_{D}, y-X_{\tau_{D}}\right)\right], \quad t>0, x, y \in \mathbb{R} . \text { (2.10) }
$$

By analogy to the free process, for any $x \in \mathbb{R}$ we define the $\lambda$-potential measure of $X_{t}^{D}$ as

$$
G_{D}^{\lambda}(x, A)=\int_{0}^{\infty} e^{-\lambda t} \mathbb{P}^{x}\left(t<\tau_{D} ; X_{t} \in A\right) d t, \quad A \in \mathcal{B}_{\mathbb{R}} .
$$

Since $p^{D}(t ; \cdot, \cdot)$ exists for all $t>0$, the $\lambda$-potential measures are absolutely continuous with the density $G_{D}^{\lambda}$ given by

$$
G_{D}^{\lambda}(x, y)=\int_{0}^{\infty} e^{-\lambda t} p^{D}(t ; x, y) d t, \quad x, y \in D .
$$

The 0-Green function is simply called the Green function and denoted by $G_{D}(x, y)$.

Next, we introduce the harmonic measure of the set $D$, which describes the distribution of $X_{\tau_{D}}$ started from $x$ on $\left\{\tau_{D}<\infty\right\}$. Namely, for any Borel $A \subset \mathbb{R}$,

$$
P_{D}(x, A)=\mathbb{P}^{x}\left(X_{\tau_{D}} \in A\right) .
$$

If the density kernel of $P_{D}$ exists then we call it the Poisson kernel for the set $D$ and denote by the same symbol $P_{D}(x, z)$. The celebrated Ikeda-Watanabe formula Ikeda and Watanabe (1962) provides a connection between the Poisson kernel and the Green function:

$$
P_{D}(x, A)=\int_{D} \nu(A-y) G_{D}(x, d y), \quad A \subset \bar{D}^{c} .
$$

Finally, we say that a Borel measurable function $u$ is harmonic in an open set $D$ with respect to $\mathbf{X}$, if for any bounded open set $B$ such that $\bar{B} \subset D$,

$$
u(x)=\mathbb{E}^{x} u\left(X_{\tau_{B}}\right), \quad x \in B .
$$


If the equality above holds also for $B=D$ then we say that $u$ is regular harmonic in $D$ (with respect to $\mathbf{X}$ ). Also, $u$ is (regular) coharmonic in $D$ if it is (regular) harmonic in $D$ with respect to the dual process $\widehat{\mathbf{X}}$. Clearly, for symmetric processes a harmonic function is coharmonic and vice versa; in general, that is not necessarily true. We remark that by the strong Markov property, a regular harmonic function is harmonic. Moreover, the Green function for the set $D$ (if it exists) is harmonic in $x$ on $D \backslash\{y\}$.

Let us note two simple observations.

Proposition 2.3. For any $x, y \in \mathbb{R}$ we have

$$
G_{\{0\}^{c}}(x, y) \leqslant H(x) \wedge H(y) .
$$

Proof: By (2.10), for any $\lambda>0$ we have

$$
\begin{aligned}
G_{\{0\}^{c}}^{\lambda}(x, y) & =u^{\lambda}(y-x)-\mathbb{E}^{x} e^{-\lambda T_{0}} u^{\lambda}\left(y-T_{0}\right) \\
& =u^{\lambda}(y-x)-u^{\lambda}(y) \frac{u^{\lambda}(-x)}{u^{\lambda}(0)} \\
& =-K^{\lambda}(y-x)+K^{\lambda}(-x)+K^{\lambda}(y)-\frac{K^{\lambda}(-x) K^{\lambda}(y)}{u^{\lambda}(0)} .
\end{aligned}
$$

Recall that $K^{\lambda} \geqslant 0$. Proceeding as in the proof of Grzywny and Ryznar (2017, Proposition 2.2) we get that $K^{\lambda}$ is subadditive on $\mathbb{R}$. Thus, $K^{\lambda}(y) \leqslant K^{\lambda}(x)+$ $K^{\lambda}(y-x)$ and consequently,

$$
G_{\{0\}^{c}}^{\lambda}(x, y) \leqslant-K^{\lambda}(y-x)+K^{\lambda}(-x)+K^{\lambda}(y) \leqslant K^{\lambda}(x)+K^{\lambda}(-x) .
$$

Similarly, we have $K^{\lambda}(-x) \leqslant K^{\lambda}(y-x)+K^{\lambda}(-y)$, and the claim follows.

Proposition 2.4. For any $|x| \in(0, R)$ we have

$$
\mathbb{E}^{x}\left[\tau_{(-R, R)} \wedge T_{0}\right] \leqslant 2 R H(x) .
$$

Proof: By Proposition 2.3,

$$
\mathbb{E}^{x}\left[\tau_{(-R, R)} \wedge T_{0}\right]=\int_{-R}^{R} G_{(-R, 0) \cup(0, R)}(x, y) d y \leqslant \int_{-R}^{R} G_{\{0\}^{c}}(x, y) d y \leqslant 2 R H(x) .
$$

2.1. Fluctuation theory. Before embarking on further results we need some introduction from the fluctuation theory of Lévy processes. The general reference here is the book Bertoin (1996).

First, let us observe that the condition (2.4) implies that $\mathbf{X}$ is of unbounded variation. Indeed, suppose the converse; then $\mathbf{X}$ can be written as a difference of two subordinators. It follows that $\operatorname{Re} \psi$ has at most linear growth and hence,

$$
\int_{0}^{\infty} \frac{d \xi}{1+\operatorname{Re} \psi(\xi)}=\infty
$$

which is a contradiction. Thus, by Rogozin (1968), 0 is regular for half-lines $(-\infty, 0)$ and $(0, \infty)$. Now, let $\mathbf{L}=\left(L_{t}: t \geqslant 0\right)$ be a local time at 0 for the process $\mathbf{S}-\mathbf{X}$, where $S_{t}=\sup _{s \leqslant t} X_{s}$, and $\mathbf{L}^{-1}$ - its right-continuous inverse, called the ascending ladder time process. Next, we define $\mathbf{H}$ by setting $H_{t}=S_{L_{t}^{-1}}=X_{L_{t}^{-1}}$ on $\left\{L_{t}^{-1}<\infty\right\}$ and $H_{t}=\infty$ otherwise. $\mathbf{H}$ is a (possibly killed) subordinator called the ascending ladder height process. 
Next, we introduce the renewal function $V$ as a potential measure of the interval $[0, x]$ for the ascending ladder height process, i.e. $V(x)=\int_{0}^{\infty} \mathbb{P}\left(H_{s} \leqslant x\right) d s$, with the convention $V(x)=0$ for $x<0$. Similarly, $\widehat{V}(x)=\int_{0}^{\infty} \mathbb{P}\left(\widehat{H}_{s} \leqslant x\right) d s$ and $\widehat{V}(x)=0$ for $x<0$. Clearly, if $\mathbf{X}$ is symmetric, then $\widehat{\mathbf{X}}=\mathbf{X}$ and consequently, $\widehat{V}=V$. Directly from the definition of $V$ and $\widehat{V}$ we conclude that both are subadditive. Moreover, by Silverstein (1980, Theorem 1 and 2), $\widehat{V}$ and $\widehat{V}^{\prime}$ are harmonic on $(0, \infty)$, and $V$ and $V^{\prime}$ are coharmonic on $(0, \infty)$. In fact, also from Silverstein (1980) we have that both $V^{\prime}$ and $\widehat{V}^{\prime}$ are positive on $(0, \infty)$, hence both $V$ and $\widehat{V}$ are actually strictly increasing on $(0, \infty)$. Note that using monotonicity and subadditivity of $\widehat{V}$, for all $\lambda \geqslant 1$ and any $r>0$ we have

$$
\widehat{V}(\lambda x) \leqslant 2 \lambda \widehat{V}(x) .
$$

One feature that will be substantial in Section 5 is the fact that the Green function for the positive half-line can be represented in terms of $V$ and $\widehat{V}$ in the following way:

$$
G_{(0, \infty)}(x, y)=\int_{0}^{x} \widehat{V}^{\prime}(u) V^{\prime}(y-x+u) d u, \quad y>x>0 .
$$

That identity is provided by Bertoin (1996, Theorem VI.20).

A number of helpful results concerning $\widehat{V}$, which is of significant importance and usage in the case of non-symmetric processes, are derived in Grzywny (2019). Below we provide a sharp estimate on the probability that the process, when exiting the interval $(0, R)$, chooses the right end. Such result seems to be interesting in and of itself, as we provide sharp two-sided bound, which is an analogue of the estimate for the symmetric case (see e.g. Grzywny and Ryznar, 2012, Proposition 3.7).

Proposition 2.5. Suppose that $\mathbb{E} X_{1}=0$ and $\operatorname{Re} \psi \in \operatorname{WLSC}(\alpha, \chi)$ for some $\alpha>1$ and $\chi \in(0,1]$. Then there is $c \in(0,1]$ such that for any $R>0$ and $0<x<R$,

$$
c \frac{\widehat{V}(x)}{\widehat{V}(R)} \leqslant \mathbb{P}^{x}\left(\tau_{(0, R)}<\tau_{(0, \infty)}\right) \leqslant \frac{\widehat{V}(x)}{\widehat{V}(R)} .
$$

Proof: Fix $R>0$ and let $x \in(0, R)$. From the proof of Grzywny (2019, Theorem $9)$ we get the following results. First,

$$
\mathbb{P}^{x}\left(\tau_{(0, R)}<\tau_{(0, \infty)}\right) \leqslant \frac{\widehat{V}(x)}{\widehat{V}(R)} .
$$

Next, we claim that there is $c_{1}$ such that

$$
c_{1} \frac{\widehat{V}(x)}{\widehat{V}\left(h^{-1}(1 / t)\right)}-\frac{\widehat{V}(x) V(R)}{t} \leqslant \mathbb{P}^{x}\left(\tau_{(0, R)}<\tau_{(0, \infty)}\right),
$$

if only $t \geqslant 1 / h(R)$. Indeed, observe that by Markov inequality and Grzywny (2019, Proposition 4),

$$
\begin{aligned}
\mathbb{P}^{x}\left(\tau_{(0, \infty)}>t\right) & \leqslant \mathbb{P}^{x}\left(\tau_{(0, R)}>t\right)+\mathbb{P}^{x}\left(\tau_{(0, R)}<\tau_{(0, \infty)}\right) \\
& \leqslant \frac{\widehat{V}(x) V(R)}{t}+\mathbb{P}^{x}\left(\tau_{(0, R)}<\tau_{(0, \infty)}\right) .
\end{aligned}
$$

Thus, using Grzywny (2019, Theorem 6) we get (2.12) for $t \geqslant 1 / h(R)$ as claimed. 
Now we specify $t>0$. Set $t=a / h(R)$, where $a \geqslant 1$. By Grzywny and Szczypkowski (2020, Lemma 2.3), there is $c_{2} \geqslant 1$ such that

$$
h^{-1}(1 / t)=h^{-1}(h(R) / a) \leqslant c_{2} a^{1 / \alpha} R .
$$

Taking into account subadditivity and monotonicity of $\widehat{V}$, we infer that

$$
\widehat{V}\left(h^{-1}(1 / t)\right) \leqslant \widehat{V}\left(c_{2} a^{1 / \alpha} R\right) \leqslant 2 c_{2} a^{1 / \alpha} \widehat{V}(R),
$$

if only $a \geqslant c_{2}^{-\alpha}$. Furthermore, by Grzywny (2019, Corollary 5), there is $c_{3} \geqslant 1$ such that

It follows that

$$
h(R) \leqslant \frac{c_{3}}{V(R) \widehat{V}(R)} .
$$

$$
\begin{aligned}
\mathbb{P}^{x}\left(\tau_{(0, R)}<\tau_{(0, \infty)}\right) & \geqslant 2^{-1} c_{1} c_{2}^{-1} a^{-1 / \alpha} \frac{\widehat{V}(x)}{\widehat{V}(R)}-\frac{c_{3}}{a} \frac{\widehat{V}(x)}{\widehat{V}(R)} \\
& =\frac{\widehat{V}(x)}{\widehat{V}(R)} \frac{1}{a}\left(2^{-1} c_{1} c_{2}^{-1} a^{(\alpha-1) / \alpha}-c_{3}\right) \geqslant \frac{1}{a} \frac{\widehat{V}(x)}{\widehat{V}(R)},
\end{aligned}
$$

if $a$ is big enough, and the claim follows.

\section{Regular variation}

In this section we aim to prove that the regular variation of the real part of the characteristic exponent implies regular variation of its imaginary part, if we impose some condition on the structure of the Lévy measure. First, we recall some basic definitions and properties from the theory of regular variation. The general reference here is the book Bingham et al. (1989).

We say that a function $f:(0, \infty) \mapsto(0, \infty)$ is regularly varying at 0 with parameter $\rho$, if for all $\lambda>0$,

$$
\lim _{x \rightarrow 0^{+}} \frac{f(\lambda x)}{f(x)}=\lambda^{\rho} .
$$

Similarly, we say that a function $f:(0, \infty) \mapsto(0, \infty)$ is regularly varying at the infinity with parameter $\rho$, if for all $\lambda>0$,

$$
\lim _{x \rightarrow \infty} \frac{f(\lambda x)}{f(x)}=\lambda^{\rho} .
$$

If $\rho=0$ then we say that a function $f$ is slowly varying at 0 (or at $\infty$ ).

Next, we recall Potter's Lemma, a not to be underestimated property of regularly varying functions (Bingham et al., 1989, Theorem 1.5.6). If a function $f$ is regularly varying at infinity with a parameter $\rho$, then for any $C>1$ and $\delta>0$ there exists $X=X(C, \delta)$, such that

$$
\frac{f(y)}{f(x)} \leqslant C \max \left[\left(\frac{y}{x}\right)^{\rho+\delta},\left(\frac{y}{x}\right)^{\rho-\delta}\right], \quad x, y>X .
$$

Let $k:(0, \infty) \mapsto \mathbb{R}$. The Mellin transform $\mathcal{M}$ of the function $k$ is defined by

$$
\mathcal{M}\{k\}(z)=\int_{0}^{\infty} t^{-z} k(t) \frac{d t}{t},
$$

for $z \in \mathbb{C}$ such that the integral converges. 
Next, for $f, g:(0, \infty) \mapsto \mathbb{R}$ we define its Mellin convolution by

$$
g \stackrel{m}{*} f(x)=\int_{0}^{\infty} g(x / t) f(t) \frac{d t}{t}, \quad x>0 .
$$

Finally, by

$$
f^{\stackrel{s}{*}} g(x)=\int_{0}^{\infty} f(x / t) d g(t), \quad x>0
$$

we denote the Mellin-Stieltjes convolution for functions $f$ and $g$ such that the Stieltjes integral is well defined.

Let us start with the observation on the equivalence between tail measure and real part of the characteristic exponent behaviour.

Proposition 3.1. Let $\alpha \in(0,2)$. Re $\psi$ is regularly varying at infinity (at 0$)$ with the exponent $\alpha$ if and only if $t \mapsto \nu(\{s:|s| \geqslant t\}$ ) is regularly varying at 0 (at infinity) with the exponent $-\alpha$. Moreover,

$$
\nu(\{s:|s| \geqslant t\}) \cong \frac{\Gamma(1+\alpha)}{\mathrm{B}\left(1-\frac{\alpha}{2}, 1+\frac{\alpha}{2}\right)} \operatorname{Re} \psi(1 / t), \quad t \rightarrow 0^{+} \quad(t \rightarrow \infty) .
$$

Proof: We compute the Laplace transform of the function Re $\psi$. By Fubini's theorem,

$$
\begin{aligned}
\mathcal{L}\{\operatorname{Re} \psi\}(\lambda) & =\int_{0}^{\infty} e^{-\lambda t} \int_{\mathbb{R}}(1-\cos t x) \nu(d x) d t=\int_{\mathbb{R}} \int_{0}^{\infty} e^{-\lambda t}(1-\cos t x) d t \nu(d x) \\
& =\frac{1}{\lambda} \int_{\mathbb{R}} \frac{x^{2}}{\lambda^{2}+x^{2}} \nu(d x) .
\end{aligned}
$$

Next,

$$
\begin{aligned}
\frac{\lambda}{2} \mathcal{L}\{\operatorname{Re} \psi\}(\lambda) & =\frac{1}{2}\left(\int_{-\infty}^{0} \frac{x^{2}}{\lambda^{2}+x^{2}} \nu(d x)+\int_{0}^{\infty} \frac{x^{2}}{\lambda^{2}+x^{2}} \nu(d x)\right) \\
& =-\int_{-\infty}^{0} \int_{x}^{0} \frac{\lambda^{2} t}{\left(\lambda^{2}+t^{2}\right)^{2}} d t \nu(d x)+\int_{0}^{\infty} \int_{0}^{x} \frac{\lambda^{2} t}{\left(\lambda^{2}+t^{2}\right)^{2}} d t \nu(d x) \\
& =-\int_{-\infty}^{0} \frac{(\lambda t)^{2}}{\left(\lambda^{2}+t^{2}\right)^{2}} \nu((-\infty, t]) \frac{d t}{t}+\int_{0}^{\infty} \frac{(\lambda t)^{2}}{\left(\lambda^{2}+t^{2}\right)^{2}} \nu([t, \infty)) \frac{d t}{t} \\
& =\int_{0}^{\infty} \frac{\left(\frac{\lambda}{t}\right)^{2}}{\left(1+\left(\frac{\lambda}{t}\right)^{2}\right)^{2}}(\nu((-\infty,-t])+\nu([t, \infty))) \frac{d t}{t}
\end{aligned}
$$

Assume that $\operatorname{Re} \psi$ varies regularly at infinity with exponent $\alpha \in(0,2)$. Let us define $\nu_{1}=\nu \mathbf{1}_{[-1,1]}$ and the characteristic exponent $\psi_{1}$ corresponding to the triplet $\left(0,0, \nu_{1}\right)$. We have $\operatorname{Re} \psi \cong \operatorname{Re} \psi_{1}$ at infinity. Indeed, since

$$
0 \leqslant \int_{|x|>1}(1-\cos (x z)) \nu(d x) \leqslant 2 \nu(\{x:|x|>1\}), \quad z \in \mathbb{R},
$$

and $\lim _{z \rightarrow \infty} \operatorname{Re} \psi(z)=\infty$, we get

$$
\frac{\operatorname{Re} \psi(z)-\operatorname{Re} \psi_{1}(z)}{\operatorname{Re} \psi(z)} \rightarrow 0
$$


as $z \rightarrow \infty$. Next, using the Abel theorem (Bingham et al., 1989, Theorem 1.7.1) one can observe that

$$
\lambda^{-1} \mathcal{L}\left\{\operatorname{Re} \psi_{1}\right\}(1 / \lambda) \cong \operatorname{Re} \psi(\lambda) \Gamma(1+\alpha), \quad \lambda \rightarrow \infty .
$$

Let $g(t)=\nu_{1}(\{s:|s| \geqslant 1 / t\}), t>0$, and $k(t)=\frac{t^{2}}{\left(1+t^{2}\right)^{2}}$. Observe that the Laplace transform of $\operatorname{Re} \psi_{1}$ is the Mellin convolution of $k$ and $g$ :

$$
\frac{1}{2 \lambda} \mathcal{L}\left\{\operatorname{Re} \psi_{1}\right\}(1 / \lambda)=\int_{0}^{\infty} k(1 /(t \lambda)) g(1 / t) \frac{d t}{t}=\int_{0}^{\infty} k(t / \lambda) g(t) \frac{d t}{t}=k^{*} g(\lambda),
$$

where in the last equality we used $k(t)=k(1 / t)$ for $t>0$.

To prove that $g(t)$ is regularly varying function, we will use Bingham et al. (1989, Theorem 4.9.1) for the function $g_{1}(t)=\int_{0}^{t} g(s) \frac{d s}{s}$ and convolution $k_{*}^{s} * g_{1}(\lambda)=k \stackrel{m}{*}$ $g(\lambda)$. Set $\sigma$ such that $-2<\sigma<-\alpha$ and $\tau=0$. Observe that

$$
\begin{aligned}
\|k\|_{\sigma, \tau} & :=\sum_{-\infty<n<\infty} \max \left(e^{-\sigma n}, e^{-\tau n}\right) \sup _{e^{n} \leqslant x \leqslant e^{n+1}}|k(x)| \\
& \leqslant \sum_{n \leqslant-1} e^{2 n}+\sum_{n \geqslant 0} \frac{e^{-\sigma n}}{e^{2 n}}<\infty .
\end{aligned}
$$

See Bingham et al. (1989, p. 210, eq. (4.4.3)) for the first appearance and introduction of the $\|k\|_{\sigma, \tau}$. Moreover, by Oberhettinger (1974, Table 1.2 (2.19)),

$$
\begin{aligned}
\mathcal{M}\{k\}(z) & =\int_{0}^{\infty} \frac{t^{2}}{\left(1+t^{2}\right)^{2}} t^{z-1} d t=\frac{1}{2} \int_{0}^{\infty} \frac{1}{(1+s)^{2}} s^{(z / 2+1)-1} d s \\
& =\frac{\Gamma\left(1-\frac{z}{2}\right) \Gamma\left(1+\frac{z}{2}\right)}{2},
\end{aligned}
$$

if only $\operatorname{Re} z \in(-2,2)$. Since $(\sigma, \tau) \subset(-2,2)$ and neither $\Gamma\left(1+\frac{z}{2}\right)$ nor $\Gamma\left(1-\frac{z}{2}\right)$ have any roots for $\operatorname{Re} z \in(\sigma, \tau)$, the Wiener condition $\mathcal{M}\{k\}(z) \neq 0$ is satisfied. Notice that $g_{1}$ is non-decreasing on $(0, \infty)$ and vanishes on $(0,1)$. Hence, $g_{1}(t)=\mathcal{O}\left(t^{\sigma}\right)$ at $0^{+}$. That is, the kernel $k$ and the function $g_{1}$ satisfy assumptions of Bingham et al. (1989, Theorem 4.9.1), hence,

$$
g_{1}(t) \cong \frac{\Gamma(1+\alpha)}{\mathrm{B}\left(1-\frac{\alpha}{2}, 1+\frac{\alpha}{2}\right)} \frac{\operatorname{Re} \psi(t)}{\alpha}, \quad t \rightarrow \infty
$$

By monotone density theorem Bingham et al. (1989, Theorem 1.7.2) and the fact that $g(1 / t) \cong \nu(\{s:|s| \geqslant t\})$ as $t$ goes to $0^{+}$, we obtain that

$$
\nu(\{s:|s| \geqslant t\}) \cong \frac{\Gamma(1+\alpha)}{\mathrm{B}\left(1-\frac{\alpha}{2}, 1+\frac{\alpha}{2}\right)} \operatorname{Re} \psi(1 / t), \quad t \rightarrow 0^{+}
$$

In particular, $t \mapsto \nu(\{s:|s| \geqslant t\})$ is regularly varying function at 0 with index $-\alpha$.

Now assume that $t \mapsto \nu(\{s:|s| \geqslant t\})$ is regularly varying function at 0 with index $-\alpha$. Again, instead of $\psi$ one can consider $\psi_{1}$. Since

$$
\operatorname{Re} \psi_{1}(z)=z \int_{0}^{1} \sin (x z) \nu(\{s:|s| \geqslant x\}) d x=\int_{0}^{z} \sin (x) \nu(\{s:|s| \geqslant x / z\}) d x
$$

one can use Potter's Lemma to justify that

$$
\lim _{z \rightarrow \infty} \frac{\operatorname{Re} \psi_{1}(z)}{\nu(\{s:|s| \geqslant 1 / z\})}=\int_{0}^{\infty} \frac{\sin x}{x^{\alpha}} d x
$$


which finishes the proof in this case.

If $\operatorname{Re} \psi$ varies regularly at 0 one can modify the above prove to obtain the behaviour of the tail of $\nu$ at infinity.

We remark that in fact, equivalence of regular variation of $\operatorname{Re} \psi$ at 0 and regular variation of the tail of $\nu$ at infinity can be easily obtained from Pitman (1968). Our proof, however, works in both cases.

If the Lévy measure is of the special form

$$
\nu(d x)=C_{d} \mathbb{1}_{\{x<0\}} \nu_{0}(d x)+C_{u} \mathbb{1}_{\{x>0\}} \nu_{0}(d x),
$$

where $\nu_{0}(d x)$ is a symmetric Lévy measure, the theorem above provides the behaviour of the one-sided tail of $\nu$ as well. For instance, if $\operatorname{Re} \psi$ is regularly varying at 0 with the exponent $\alpha \in(0,2)$, then

$$
\nu_{0}([t, \infty)) \cong \frac{1}{C_{u}+C_{d}} \frac{\Gamma(1+\alpha)}{\mathrm{B}\left(1-\frac{\alpha}{2}, 1+\frac{\alpha}{2}\right)} \operatorname{Re} \psi(1 / t), \quad t \rightarrow \infty .
$$

This is the case for stable processes, where even the equality holds true.

Lemma 3.2. If $\operatorname{Re} \psi$ varies regularly at 0 with an exponent $\alpha>1$, then

$$
\int_{(-1,1)^{c}}|x| \nu(d x)<\infty .
$$

Proof: If $\operatorname{Re} \psi$ varies regularly at 0 with a positive exponent then $\operatorname{Re} \psi \cong \psi^{*}$ near 0 . Hence, $h(r) \approx \operatorname{Re} \psi(1 / r)$ for large $r>R_{0}$. By the Potter Lemma we get

$$
\begin{aligned}
\int_{|x|>1}|x| \nu(d x) & =\int_{0}^{\infty} \nu(\{s:|s|>1 \vee u\}) d u \\
& \leqslant \int_{0}^{\infty} h(1 \vee u) d u \leqslant h(1) R_{0}+c \int_{R_{0}}^{\infty} \operatorname{Re} \psi(1 / u) d u<\infty .
\end{aligned}
$$

The claim follows immediately.

Lemma 3.3. Assume that $f(s), s \geqslant 0$, is a function which is non-negative, regularly varying at $\infty$ with parameter $-\alpha$, where $\alpha \in(1,2)$, and such that $\int_{0}^{\infty}(1 \wedge$ $\left.s^{2}\right) f(s) d s<\infty$. Then the transformation

$$
x \mapsto \int_{0}^{\infty}(1-\cos x s) f(s) d s, \quad x \geqslant 0,
$$

is regularly varying at 0 with the parameter $\alpha-1$ and satisfies

$$
\int_{0}^{\infty}(1-\cos x s) f(s) d s \cong-\frac{f(1 / x)}{x} \frac{\pi}{2 \Gamma(\alpha) \cos \left(\frac{\pi \alpha}{2}\right)}, \quad x \rightarrow 0^{+} .
$$

Proof: Let $x>0$. By the Potter Lemma, one can set $A$ such that for $s>A$, there exist $-\alpha<-\hat{\alpha}<-1$ and $M$ such that $\frac{f(s / x)}{f(1 / x)}<M s^{-\hat{\alpha}}$. Notice that

$$
\begin{aligned}
& \frac{x}{f(1 / x)} \int_{0}^{\infty}(1-\cos x s) f(s) d s \\
& =\frac{x}{f(1 / x)} \int_{0}^{A}(1-\cos x s) f(s) d s+\frac{x}{f(1 / x)} \int_{A}^{\infty}(1-\cos x s) f(s) d s \\
& =\frac{x}{f(1 / x)} \int_{0}^{A}(1-\cos x s) f(s) d s+\int_{A x}^{\infty}(1-\cos s) \frac{f(s / x)}{f(1 / x)} d s .
\end{aligned}
$$


Again by the Potter Lemma, for constant $C$ and $\rho<2$ there exists $\varepsilon>0$ such that $C x^{\rho} \leqslant f(1 / x)$, when $0<x<\varepsilon$. Then we can observe that

$$
\frac{x}{f(1 / x)} \int_{0}^{A}(1-\cos x s) f(s) d s \leqslant C \frac{x^{3}}{x^{\rho}} \int_{0}^{A} s^{2} f(s) d s \rightarrow 0,
$$

as $x \rightarrow 0^{+}$. Moreover, for $|s|>A x$,

$$
(1-\cos s) \frac{f(s / x)}{f(1 / x)} \leqslant M(1-\cos s) s^{-\hat{\alpha}} .
$$

It allows us to make use of the dominated convergence theorem. Therefore,

$$
\begin{aligned}
\lim _{x \rightarrow \infty} \frac{x}{f(1 / x)} \int_{0}^{\infty}(1-\cos x s) f(s) d s & =\int_{0}^{\infty}(1-\cos s) s^{-\alpha} d s=\frac{\pi}{2 \Gamma(\alpha) \sin \left(\frac{\pi(\alpha-1)}{2}\right)} \\
& =-\frac{\pi}{2 \Gamma(\alpha) \cos \left(\frac{\pi \alpha}{2}\right)},
\end{aligned}
$$

where the second equality follows from Sato (1999, Theorem 14.15).

Next theorem is the main result of this section. It shows that under assumption (3.2), regular variation of the real part of the Lévy exponent implies regular variation of the imaginary part. This is the case for instance for spectrally one-sided Lévy processes.

Theorem 3.4. Assume that the Lévy measure $\nu(d x)$ satisfies (3.2). Let the real part of the characteristic exponent $\operatorname{Re} \psi(\xi)$ be regularly varying at 0 with a parameter $\alpha \in(1,2)$. If $\gamma_{1}=\gamma-\int_{(-1,1)^{c}} x \nu(d x)=0$ then the imaginary part $\operatorname{Im} \psi(\xi)$ satisfies

$$
\operatorname{Im} \psi(\xi) \cong-\frac{C_{u}-C_{d}}{C_{u}+C_{d}} \tan \left(\frac{\pi \alpha}{2}\right) \operatorname{Re} \psi(\xi), \quad \xi \rightarrow 0^{+} .
$$

For $\gamma_{1} \neq 0$ we have

$$
\operatorname{Im} \psi(\xi) \cong \gamma_{1} \xi, \quad \xi \rightarrow 0 .
$$

Proof: By Proposition 3.1, $t \mapsto \nu_{0}([t, \infty))$ is a regularly varying function with the exponent $-\alpha$ at $\infty$. More precisely,

$$
\nu_{0}([t, \infty)) \cong \frac{1}{C_{u}+C_{d}} \frac{\Gamma(1+\alpha)}{\mathrm{B}\left(1-\frac{\alpha}{2}, 1+\frac{\alpha}{2}\right)} \operatorname{Re} \psi(1 / t), \quad t \rightarrow \infty .
$$

By Lemma 3.2 we know that $\int_{(-1,1)^{c}}|x| \nu(d x)<\infty$. We are therefore allowed to use the representation (2.3) of the imaginary part of the function $\psi$. Let $\xi>0$. Observe that

$$
\begin{aligned}
\operatorname{Im} \psi(\xi) & =\gamma_{1} \xi+\int_{\mathbb{R}}(\xi x-\sin \xi x) \nu(d x) \\
& =\gamma_{1} \xi+\left(C_{u}-C_{d}\right) \int_{0}^{\infty} \int_{0}^{x}(\xi t-\sin \xi t)^{\prime} d t \nu_{0}(d x) \\
& =\gamma_{1} \xi+\left(C_{u}-C_{d}\right) \xi \int_{0}^{\infty}(1-\cos \xi t) \int_{t}^{\infty} \nu_{0}(d x) d t \\
& =\gamma_{1} \xi+\left(C_{u}-C_{d}\right) \xi \int_{0}^{\infty}(1-\cos \xi t) \nu_{0}([t, \infty)) d t .
\end{aligned}
$$


Let $C_{u} \neq C_{d}$. Observe that $f(s)=\nu([s, \infty))$ satisfies the assumptions of Lemma 3.3. Thus, a function $\xi \mapsto\left(C_{u}-C_{d}\right) \xi \int_{0}^{\infty}(1-\cos \xi t) \nu_{0}([t, \infty)) d t$ is also regularly varying function at 0 with the exponent $\alpha$. Assume that $\gamma_{1} \neq 0$. Then

$$
\frac{\gamma_{1} \xi+\xi\left(C_{u}-C_{d}\right) \int_{0}^{\infty}(1-\cos \xi t) \nu_{0}([t, \infty)) d t}{\gamma_{1} \xi} \rightarrow 1
$$

as $\xi \rightarrow 0^{+}$, which follows from the Potter Lemma for the function $\left(C_{u}-C_{d}\right) \xi \int_{0}^{\infty}(1-$ $\cos \xi t) \nu_{0}([t, \infty)) d t$. Then $\operatorname{Im} \psi(\xi)$ is comparable at zero with a linear function. Now, assume that $\gamma_{1}=0$. Again by Lemma 3.3, if $\xi \rightarrow 0^{+}$,

$$
\xi \int_{0}^{\infty}(1-\cos \xi t) \nu_{0}([t, \infty)) d t \cong \nu_{0}([1 / \xi, \infty)) \frac{\pi}{2 \Gamma(\alpha) \cos \left(\frac{\pi \alpha}{2}\right)}
$$

Using $\Gamma(1-z) \Gamma(z)=\frac{\pi}{\sin \pi z}$ and invoking (3.3), we obtain

$$
\begin{aligned}
\operatorname{Im} \psi(\xi) & \cong-\frac{C_{u}-C_{d}}{C_{u}+C_{d}} \frac{\alpha \Gamma(\alpha)}{\Gamma\left(1-\frac{\alpha}{2}\right) \Gamma\left(1+\frac{\alpha}{2}\right)} \frac{\pi}{2 \Gamma(\alpha) \cos \left(\frac{\pi \alpha}{2}\right)} \operatorname{Re} \psi(\xi) \\
& \cong-\frac{C_{u}-C_{d}}{C_{u}+C_{d}} \frac{\frac{\alpha}{2} \Gamma\left(\frac{\alpha}{2}\right)}{\Gamma\left(\frac{\alpha}{2}\right) \Gamma\left(1-\frac{\alpha}{2}\right) \Gamma\left(1+\frac{\alpha}{2}\right)} \frac{\pi}{\cos \left(\frac{\pi \alpha}{2}\right)} \operatorname{Re} \psi(\xi) \\
& \cong-\frac{C_{u}-C_{d}}{C_{u}+C_{d}} \tan \left(\frac{\pi \alpha}{2}\right) \operatorname{Re} \psi(\xi), \quad \xi \rightarrow 0^{+} .
\end{aligned}
$$

\section{Asymptotics}

If the process $X_{t}$ is symmetric then by Yano (2013, Theorem 4.2), the function $K$ is well-defined. Furthermore, for non-symmetric case by Yano (2013, Proposition 6.1), existence of first derivatives of $(\operatorname{Re} \psi(\xi))^{\prime}$ and $(\operatorname{Im} \psi(\xi))^{\prime}$ such that

$$
\int_{0}^{\infty} \frac{\left(\left|(\operatorname{Re} \psi(\xi))^{\prime}\right|+\left|(\operatorname{Im} \psi(\xi))^{\prime}\right|\right)\left(\xi^{2} \wedge 1\right)}{|\psi(\xi)|^{2}} d \xi<\infty .
$$

is sufficient. We remark here that in view of Sato (1999, Theorem 21.9) and discussion at the beginning of Subsection 2.1, the condition (L1') in Yano (2013) always implies (L2).

Unfortunately, (4.1) does not suit our case and therefore, we prove the existence of $K$ in several cases.

Lemma 4.1. Assume that $1 /(1+\operatorname{Re} \psi)$ is integrable and $\operatorname{Im} \psi \geqslant 0$ on $(0, \varepsilon)$ for some $\varepsilon>0$. Then $K$ exists and

$$
K(x)=\frac{1}{\pi} \int_{0}^{\infty} \operatorname{Re}\left[\frac{1}{\psi(s)}\left(1-e^{-i x s}\right)\right] d s .
$$

Proof: Since $e^{-x} \leqslant(1+x)^{-1}, x \geqslant 0$ we obtain $e^{-\psi} \in L^{1}(\mathbb{R})$. By the RiemannLebesgue Lemma we have $\operatorname{Re} \psi(\xi) \rightarrow \infty$ as $\xi \rightarrow \infty$. Since $\operatorname{Re} \psi(\xi)>0$ for $\xi \neq 0$, that implies $1 / \operatorname{Re} \psi \in L^{1}([\delta, \infty))$ for any $\delta>0$. Next, let us observe that $\operatorname{Re} \psi(\xi) \geqslant$ $c \xi^{2},|\xi| \leqslant 1$, for some $c>0$. Hence,

$$
\int_{\mathbb{R}} \frac{1-\cos (x \xi)}{\operatorname{Re} \psi(\xi)} d \xi<\infty
$$


By the dominated convergence theorem, for $x \in \mathbb{R}$,

$$
\lim _{\lambda \rightarrow 0^{+}} \int_{\mathbb{R}} \frac{(1-\cos (x \xi))(\lambda+\operatorname{Re} \psi(\xi))}{|\lambda+\psi(\xi)|^{2}} d \xi=\int_{\mathbb{R}} \frac{(1-\cos (x \xi)) \operatorname{Re} \psi(\xi)}{|\psi(\xi)|^{2}} d \xi,
$$

and

$$
\lim _{\lambda \rightarrow 0^{+}} \int_{|\xi| \geqslant \varepsilon \wedge(\pi /|x|)} \frac{\sin (x \xi) \operatorname{Im} \psi(\xi)}{|\lambda+\psi(\xi)|^{2}} d \xi=\int_{|\xi| \geqslant \varepsilon \wedge(\pi /|x|)} \frac{\sin (x \xi) \operatorname{Im} \psi(\xi)}{|\psi(\xi)|^{2}} d \xi .
$$

For $|\xi|<\varepsilon \wedge(\pi /|x|)$, the function $\xi \mapsto \sin (x \xi) \operatorname{Im} \psi(\xi)$ is non-negative, therefore by the monotone convergence theorem,

$$
\lim _{\lambda \rightarrow 0^{+}} \int_{|\xi|<\varepsilon \wedge(\pi /|x|)} \frac{\sin (x \xi) \operatorname{Im} \psi(\xi)}{|\lambda+\psi(\xi)|^{2}} d \xi=\int_{|\xi|<\varepsilon \wedge(\pi /|x|)} \frac{\sin (x \xi) \operatorname{Im} \psi(\xi)}{|\psi(\xi)|^{2}} d \xi .
$$

Since $0 \leqslant K^{\lambda}(x) \leqslant H(x)<\infty$ for every $\lambda>0$ and $x \in \mathbb{R}$ the above integral is finite. Finally let us notice that the integrand is an even function which ends the proof.

Corollary 4.2. If $1 /(1+\operatorname{Re} \psi)$ is integrable, $\mathbb{E} X_{1}$ exists and $\mathbb{E} X_{1} \neq 0$ then $K$ is well-defined and (4.2) holds.

Proof: Since $\mathbb{E}\left|X_{1}\right|<\infty$, we have

$$
\psi(\xi)=\sigma^{2} \xi^{2}+i \gamma_{1} \xi+\int_{\mathbb{R}}\left(1+i \xi z-e^{i \xi z}\right) \nu(d z) .
$$

A consequence of the dominated convergence theorem is

$$
\lim _{\xi \rightarrow 0^{+}} \frac{\operatorname{Im} \psi(\xi)}{\xi}=\gamma_{1}
$$

Hence, if $\gamma_{1}=\mathbb{E} X_{1} \neq 0$ then $\operatorname{Im} \psi$ has a constant sign on $(0, \varepsilon)$ for some $\varepsilon>0$, which finishes the proof due to Lemma 4.1.

Proposition 4.3. Assume that $1 /(1+\operatorname{Re} \psi)$ is integrable and there is $c>0$ such that

$$
\int_{|z|>r}|z| \nu(d z) \leqslant \operatorname{crh}(r), \quad r>1 .
$$

Then $K$ exists and (4.2) holds.

Remark 4.4. If $\operatorname{Re} \psi \in \operatorname{WLSC}(\alpha, \chi)$ for some $\alpha>1$ and $\chi \in(0,1]$, then the assumptions of Proposition 4.3 are satisfied.

Proof: By (4.4) we have $\mathbb{E}\left|X_{1}\right|<\infty$. If $\mathbb{E} X_{1} \neq 0$ we apply Corollary 4.2 to get the claim of the Proposition. Therefore, assume that $\mathbb{E} X_{1}=0$ and then

$$
\psi(\xi)=\sigma^{2} \xi^{2}+\int_{\mathbb{R}}\left(1+i \xi z-e^{i \xi z}\right) \nu(d z) .
$$

By the proof of Lemma 4.1 it is enough to prove that (4.3) holds. Let us consider a Lévy measure $\tilde{\nu}(d x)=1_{(0,1)}(x) x^{-5 / 2} d x+1_{[1, \infty)} \nu(d x)$ and a characteristic exponent

$$
\tilde{\psi}(\xi)=\int_{0}^{\infty}\left(1+i \xi z-e^{i \xi z}\right) \tilde{\nu}(d z), \quad \xi \in \mathbb{R} .
$$

Since $\operatorname{Re} \tilde{\psi}(\xi) \approx|\xi|^{3 / 2},|\xi| \geqslant 1$, and

$$
\operatorname{Im} \tilde{\psi}(\xi)=\int^{\infty}(\xi z-\sin (\xi z)) \tilde{\nu}(d z) \geqslant 0
$$


we can apply Lemma 4.1 and its proof to obtain finiteness of

$$
\int_{0}^{\infty} \frac{|\sin (x \xi) \operatorname{Im} \tilde{\psi}(\xi)|}{|\tilde{\psi}(\xi)|^{2}} d \xi, \quad x \in \mathbb{R} .
$$

Let

$$
\psi_{1}(\xi)=\int_{0}^{\infty}\left(1+i \xi z-e^{i \xi z}\right) \nu(d z),
$$

and $\psi_{2}(\xi)=\psi(\xi)-\psi_{1}(\xi)$. Notice that $\operatorname{Re} \psi_{1} \approx \operatorname{Re} \tilde{\psi}$ and $\operatorname{Im} \psi_{1} \approx \operatorname{Im} \tilde{\psi}$ on $(0,1)$ and $\operatorname{Im} \psi_{1}(\xi), \operatorname{Im} \psi_{2}(-\xi) \geqslant 0, \xi \geqslant 0$. Hence,

$$
\int_{0}^{1} \frac{\left|\sin (x \xi) \operatorname{Im} \psi_{1}(\xi)\right|}{\left|\psi_{1}(\xi)\right|^{2}} d \xi<\infty .
$$

But we also have that $\left|\operatorname{Im} \psi_{1}(\xi)\right| \leqslant c \operatorname{Re} \psi(\xi)$ for $|\xi|<1$. Indeed, by Taylor's formula and (4.4),

$$
\begin{aligned}
\left|\operatorname{Im} \psi_{1}(\xi)\right| & \lesssim \int_{0}^{1 /|\xi|}(|\xi| z)^{3} \nu(d z)+|\xi| \int_{1 /|\xi|}^{\infty} z \nu(d z) \\
& \lesssim|\xi|^{2} \int_{0}^{1 /|\xi|} z^{2} \nu(d z)+|\xi| \int_{1 /|\xi|}^{\infty} z \nu(d z) \\
& \lesssim|\xi|^{2} \int_{0}^{1 /|\xi|} z^{2} \nu(d z)+h(1 /|\xi|) \\
& \lesssim \operatorname{Re} \psi(\xi)
\end{aligned}
$$

where the last inequality follows from (2.9) and Grzywny and Szczypkowski (2020, Lemma 2.3). These implies

$$
\int_{0}^{1} \frac{\left|\sin (x \xi) \operatorname{Im} \psi_{1}(\xi)\right|}{|\psi(\xi)|^{2}} d \xi<\infty .
$$

Hence, by the monotone convergence theorem,

$$
\lim _{\lambda \rightarrow 0^{+}} \int_{|\xi|<\varepsilon \wedge(\pi /|x|)} \frac{\sin (x \xi) \operatorname{Im} \psi_{1}(\xi)}{|\lambda+\psi(\xi)|^{2}} d \xi=\int_{|\xi|<\varepsilon \wedge(\pi /|x|)} \frac{\sin (x \xi) \operatorname{Im} \psi_{1}(\xi)}{|\psi(\xi)|^{2}} d \xi,
$$

and the limit is finite. Again by the monotone convergence theorem,

$$
\lim _{\lambda \rightarrow 0^{+}} \int_{|\xi|<\varepsilon \wedge(\pi /|x|)} \frac{\sin (x \xi) \operatorname{Im} \psi_{2}(\xi)}{|\lambda+\psi(\xi)|^{2}} d \xi=\int_{|\xi|<\varepsilon \wedge(\pi /|x|)} \frac{\sin (x \xi) \operatorname{Im} \psi_{2}(\xi)}{|\psi(\xi)|^{2}} d \xi .
$$

Combining the above limits together we obtain (4.3), which ends the proof.

Corollary 4.5. Let $\operatorname{Re} \psi$ vary regularly at 0 with an exponent $\alpha \in(1,2]$, then $K$ is well-defined and (4.2) holds.

Now we turn our attention to the asymptotic behaviour of the tail of the distribution of the first hitting time.

Lemma 4.6. Assume that $\operatorname{Re} \psi$ varies regularly at 0 with an exponent $\alpha \in(1,2]$ and

$$
\frac{\operatorname{Im} \psi(\xi)}{\operatorname{Re} \psi(\xi)} \rightarrow C_{I}, \quad \xi \rightarrow 0^{+}
$$

for some $C_{I} \in \mathbb{R}$. Then

$$
\lambda u^{\lambda}(0) \cong(\operatorname{Re} \psi)^{-1}(\lambda) C\left(\alpha, C_{I}\right), \quad \lambda \rightarrow 0^{+},
$$


where

$$
C\left(\alpha, C_{I}\right)=\frac{\cos \left(\operatorname{arctg}\left(C_{I}\right) / \alpha\right)}{\alpha\left(1+C_{I}^{2}\right)^{1 /(2 / \alpha)} \sin (\pi / \alpha)} .
$$

Proof: Denote $\theta(\xi):=\operatorname{Re} \psi(\xi)$ and $\omega(\xi):=\operatorname{Im} \psi(\xi)$. We have

$$
u^{\lambda}(0)=\frac{1}{\pi} \int_{0}^{\infty} \frac{\lambda+\theta(\xi)}{(\lambda+\theta(\xi))^{2}+\omega(\xi)^{2}} d \xi
$$

Notice that, for any $\delta>0$,

$$
\mathrm{I}_{1}(\lambda):=\int_{\delta}^{\infty} \frac{\lambda+\theta(\xi)}{(\lambda+\theta(\xi))^{2}+\omega(\xi)^{2}} d \xi \leqslant \int_{\delta}^{\infty} \frac{1}{\theta(\xi)} d \xi<\infty .
$$

Since $\alpha>1$, we obtain $\frac{\lambda}{\theta^{-1}(\lambda)} \mathrm{I}_{1}(\lambda) \rightarrow 0$ as $\lambda \rightarrow 0^{+}$, hence it does not have impact on the asymptotic behaviour.

Set $\mathrm{I}_{2}(\lambda):=\pi u^{\lambda}(0)-\mathrm{I}_{1}(\lambda)$. Since $\theta$ is continuous function we have $\theta\left(\theta^{-1}(s)\right)=s$. Hence,

$$
\begin{aligned}
\frac{\lambda}{\theta^{-1}(\lambda)} \mathrm{I}_{2}(\lambda) & =\int_{0}^{\frac{\delta}{\theta^{-1}(\lambda)}} \frac{1+\theta\left(\theta^{-1}(\lambda) w\right) / \lambda}{\left(1+\theta\left(\theta^{-1}(\lambda) w\right) / \lambda\right)^{2}+\left(\omega\left(\theta^{-1}(\lambda) w\right) / \lambda\right)^{2}} d w \\
& =\int_{0}^{\frac{\delta}{\theta^{-1}(\lambda)}} \frac{1+\frac{\theta\left(\theta^{-1}(\lambda) w\right)}{\theta\left(\theta^{-1}(\lambda)\right)}}{\left.\left(1+\frac{\theta\left(\theta^{-1}(\lambda) w\right)}{\theta\left(\theta^{-1}(\lambda)\right)}\right)^{2}+\left(\frac{\omega(\theta-1}{\theta\left(\theta^{-1}(\lambda) w\right)}\right) \frac{\theta\left(\theta^{-1}(\lambda) w\right)}{\theta\left(\theta^{-1}(\lambda)\right)}\right)^{2}} d w .
\end{aligned}
$$

Now we will choose $\delta$. Set $\rho$ such that $1<\rho<\alpha$. By the Potter Lemma there exists $\delta>0$ such that for $\lambda<\theta(\delta), s>1$ and $\theta^{-1}(\lambda) s<\delta$,

$$
\frac{\theta\left(\theta^{-1}(\lambda) s\right)}{\theta\left(\theta^{-1}(\lambda)\right)} \geqslant \frac{1}{2} s^{\rho}
$$

The integrand in (4.5) is then dominated by

$$
\frac{1}{1+\frac{\theta\left(\theta^{-1}(\lambda) w\right)}{\theta\left(\theta^{-1}(\lambda)\right)}} \leqslant \frac{2}{1+w^{\rho} / 2}, \quad w \leqslant \delta / \theta(\lambda) .
$$

By the dominated convergence theorem,

$$
\lim _{\lambda \rightarrow 0^{+}} \frac{\lambda}{\theta^{-1}(\lambda)} u^{\lambda}(0)=\frac{1}{\pi} \int_{0}^{\infty} \frac{1+w^{\alpha}}{\left(1+w^{\alpha}\right)^{2}+\left(C_{I} w^{\alpha}\right)^{2}} d w,
$$

which ends the proof since the limit is equal to $u^{1}(0)$ for stable processes (see Port, 1967, p. 389).

Theorem 4.7. Assume that $\operatorname{Re} \psi(\xi)$ varies regularly at 0 with an exponent $\alpha \in$ $(1,2]$ and $\lim _{\xi \rightarrow 0^{+}} \operatorname{Im} \psi(\xi) / \operatorname{Re} \psi(\xi)=C_{I}$. Let $B$ be a compact set such that $0 \in B$. Then, for $x \in \mathbb{R}$,

$$
\lim _{t \rightarrow \infty} t(\operatorname{Re} \psi)^{-1}(1 / t) \mathbb{P}^{x}\left(T_{B}>t\right)=\frac{1}{C\left(\alpha, C_{I}\right) \Gamma(1 / \alpha)}\left(K(-x)-\mathbb{E}^{x} K\left(-X_{T_{B}}\right)\right) .
$$

Let us note here that Theorem 4.7 extends Port (1967, Theorem 2), where general recurrent stable processes are treated.

Proof: We have

$$
\mathcal{L}\left(\mathbb{P}^{x}\left(T_{B}>\cdot\right)\right)(\lambda)=\frac{1}{\lambda}\left[1-\mathbb{E}^{x} e^{-\lambda T_{B}}\right]
$$


In view of Proposition 2.3 and the fact that $0 \leqslant G_{B^{c}}^{\lambda}(x, 0) \leqslant G_{\{0\}^{c}}(x, 0)$, we can observe that $G_{B^{c}}^{\lambda}(x, 0)=0$. Hence,

$$
\begin{aligned}
\lambda u^{\lambda}(0) \mathcal{L}\left(\mathbb{P}^{x}\left(T_{B}>\cdot\right)\right)(\lambda) & =u^{\lambda}(0)-u^{\lambda}(-x)+u^{\lambda}(-x) \\
& -\mathbb{E}^{x} e^{-\lambda T_{B}}\left(u^{\lambda}(0)-u^{\lambda}\left(-X_{T_{B}}\right)\right)-\mathbb{E}^{x} e^{-\lambda T_{B}}\left(u^{\lambda}\left(-X_{T_{B}}\right)\right) \\
& =K^{\lambda}(-x)-\mathbb{E}^{x} e^{-\lambda T_{B}} K^{\lambda}\left(-X_{T_{B}}\right)+G_{B^{c}}^{\lambda}(x, 0) \\
& =K^{\lambda}(-x)-\mathbb{E}^{x} e^{-\lambda T_{B}} K^{\lambda}\left(-X_{T_{B}}\right) .
\end{aligned}
$$

Since $K^{\lambda}$ is bounded by $H$ and, by Proposition 2.2, $H$ is bounded on $B$ because of its compactness, using the dominated convergence theorem, Lemma 4.6 and Corollary 4.5 we infer that

$$
\lim _{\lambda \rightarrow 0^{+}}(\operatorname{Re} \psi)^{-1}(\lambda) \mathcal{L}\left(\mathbb{P}^{x}\left(T_{B}>\cdot\right)\right)(\lambda)=\left(K(-x)-\mathbb{E}^{x} K\left(-X_{T_{B}}\right)\right) / C\left(\alpha, C_{I}\right) .
$$

Let $U(s):=\int_{0}^{s} \mathbb{P}^{x}\left(T_{B}>t\right) d t$. We have

$$
\mathcal{L} U(\lambda)=\frac{1}{\lambda} \mathcal{L}\left(\mathbb{P}^{x}\left(T_{B}>\cdot\right)\right)(\lambda)
$$

Hence,

$$
\lim _{\lambda \rightarrow 0^{+}} \lambda(\operatorname{Re} \psi)^{-1}(\lambda) \mathcal{L} U(\lambda)=\left(K(-x)-\mathbb{E}^{x} K\left(-X_{T_{B}}\right)\right) / C\left(\alpha, C_{I}\right) .
$$

Notice that $(\operatorname{Re} \psi)^{-1}$ is regularly varying with an exponent $1 / \alpha$, thus, by the Tauberian theorem Bingham et al. (1989, Theorem 1.7.1) we can observe that

$$
\lim _{t \rightarrow \infty}(\operatorname{Re} \psi)^{-1}(1 / t) U(t)=\frac{1}{C\left(\alpha, C_{I}\right) \Gamma(1+1 / \alpha)}\left(K(-x)-\mathbb{E}^{x} K\left(-X_{T_{B}}\right)\right) .
$$

Eventually, by the monotone density theorem (Bingham et al., 1989, Theorem 1.7.2),

$$
\lim _{t \rightarrow \infty} t(\operatorname{Re} \psi)^{-1}(1 / t) \mathbb{P}^{x}\left(T_{B}>t\right)=\frac{1}{\alpha C\left(\alpha, C_{I}\right) \Gamma(1+1 / \alpha)}\left(K(-x)-\mathbb{E}^{x} K\left(-X_{T_{B}}\right)\right) .
$$

Since $\mathbb{E}^{x} K\left(X_{T_{0}}\right)=0$, we obtain the following Corollary.

Corollary 4.8. Assume that $\operatorname{Re} \psi$ varies regularly at 0 with an exponent $\alpha \in(1,2]$ and suppose that $\lim _{\xi \rightarrow 0^{+}} \operatorname{Im} \psi(\xi) / \operatorname{Re} \psi(\xi)=C_{I}$. Then, for $x \in \mathbb{R}$,

$$
\lim _{t \rightarrow \infty} t(\operatorname{Re} \psi)^{-1}(1 / t) \mathbb{P}^{x}\left(T_{0}>t\right)=\frac{1}{C\left(\alpha, C_{I}\right) \Gamma(1 / \alpha)} K(-x) .
$$

Using Theorem 3.4 we conclude the asymptotic behaviour for Lévy measures of specific type (3.2).

Corollary 4.9. Suppose that $\nu(d x)$ is of the form (3.2). Assume that $\mathbb{E} X_{1}=0$ and $\operatorname{Re} \psi$ is regularly varying at 0 with parameter $\alpha \in(1,2)$. Then (4.8) holds true. In particular, this is the case for spectrally one-sided Lévy processes.

Proposition 4.10. Suppose that $1 /(1+\operatorname{Re} \psi)$ is integrable and $\mathbb{E} X_{1}$ exists. If $\mathbb{E} X_{1} \neq 0$, then

$$
\mathbb{P}^{x}\left(T_{B}>t\right) \cong\left(K(-x)-\mathbb{E}^{x} K\left(-X_{T_{B}}\right)\right) \kappa, \quad t \rightarrow \infty,
$$

where $\kappa$ is as in (2.6). 
Proof: Let us observe that by Sato (1999, Theorem 36.7), we have $\kappa>0$. Hence, by (4.7), Corollary 4.2 and the Tauberian theorem Bingham et al. (1989, Theorem 1.7.1) we obtain the claim.

Corollary 4.11. Under the assumptions of the above proposition the compensated kernel exists and it is coharmonic on $(0, \infty)$.

\section{Harnack inequality and boundary behaviour}

This Section is devoted to the proof of the Harnack inequality and a discussion on its consequences. The main result here is Theorem 5.4, which will then allow us to deduce some useful properties, including boundary behaviour of harmonic functions.

Lemma 5.1. Suppose that $\mathbb{E} X_{1}=0$ and $\operatorname{Re} \psi \in \operatorname{WLSC}(\alpha, \chi)$ for some $\alpha>1$ and $\chi \in(0,1]$. Then there are $\delta_{1} \in(0,1]$ and $c>0$, depending only on the scalings, such that for any $R>0$,

$$
G_{(-R, R)}(x, y) \geqslant c H(R), \quad|x|,|y| \leqslant \delta_{1} R .
$$

Proof: By the sweeping formula, for any $\lambda>0$ and any $x, y \in \mathbb{R}$ we have

$$
G_{(-R, R)}(x, y) \geqslant G_{(-R, R)}^{\lambda}(x, y)=U^{\lambda}(y-x)-\mathbb{E}^{x} e^{-\lambda \tau_{(-R, R)}} U^{\lambda}\left(y-X_{\tau_{(-R, R)}}\right) .
$$

Since $\mathbb{E} X_{1}=0$, we have $b_{r}=-\int_{|z| \geqslant r} z \nu(d z)$, thus, by Grzywny and Szczypkowski (2020, Lemma 2.10), there is $c_{1} \in(0,1]$ such that $t\left|b_{h^{-1}(1 / t)}\right| \leqslant c_{1} h^{-1}(1 / t)$ for all $t>0$. Hence, by Grzywny and Szczypkowski (2020, Theorem 5.4) with $\theta=$ $\left(2+c_{1}\right) h^{-1}(\lambda)$, there is $c_{2} \in(0,1]$ such that for all $|x|,|y|<h^{-1}(\lambda)$,

$$
U^{\lambda}(y-x) \geqslant \int_{1 / \lambda}^{\infty} e^{-\lambda t} p(t, y-x) d t \geqslant c_{2} \int_{1 / \lambda}^{\infty} e^{-\lambda t} \frac{d t}{h^{-1}(1 / t)}
$$

By Grzywny and Szczypkowski (2020, Lemma 2.3), there is $c_{3} \in(0,1]$ such that

$$
U^{\lambda}(y-x) \geqslant \frac{c_{2} c_{3}}{\lambda h^{-1}(\lambda)} \int_{1}^{\infty} e^{-s} \frac{d s}{s^{1 / \alpha}}=c_{4} \frac{1}{\lambda h^{-1}(\lambda)},
$$

with $c_{4}=c_{2} c_{3} / \int_{1}^{\infty} e^{-s} s^{-1 / \alpha} d s$.

Next, using the estimate on the supremum of the density $p(t, \cdot)$ (see Grzywny and Szczypkowski, 2020, Theorem 3.1) we infer that

$$
\mathbb{E}^{x} e^{-\lambda \tau_{(-R, R)}} U^{\lambda}\left(y-X_{\tau_{(-R, R)}}\right) \leqslant \mathbb{E}^{x} e^{-\lambda \tau_{(-R, R)}} \int_{0}^{\infty} e^{-\lambda t} \frac{d t}{h^{-1}(1 / t)} .
$$

By the scaling property of $h^{-1}$,

$$
\int_{0}^{1 / \lambda} e^{-\lambda t} \frac{d t}{h^{-1}(1 / t)} \lesssim \frac{1}{\lambda^{1 / \alpha} h^{-1}(\lambda)} \int_{0}^{1 / \lambda} \frac{d t}{t^{1 / \alpha}}=c_{\alpha} \frac{1}{\lambda h^{-1}(\lambda)} .
$$

Moreover, by monotonicity of $h^{-1}$,

$$
\int_{1 / \lambda}^{\infty} e^{-\lambda t} \frac{d t}{h^{-1}(1 / t)} \leqslant \frac{1}{h^{-1}(\lambda)} \int_{1 / \lambda}^{\infty} e^{-\lambda t} d t=e^{-1} \frac{1}{\lambda h^{-1}(\lambda)} .
$$

Now let $t_{0}>0$. By Pruitt's estimate (see Pruitt, 1981), there is $c_{5}>0$ such that

$$
\mathbb{E}^{x}\left[\tau_{(-R, R)} \leqslant t_{0} ; e^{-\lambda \tau_{(-R, R)}}\right] \leqslant c_{5} t_{0}\left(h(R)+R^{-1}\left|b_{R}\right|\right) .
$$


Furthermore,

$$
\mathbb{E}^{x}\left[\tau_{(-R, R)} \geqslant t_{0} ; e^{-\lambda \tau_{(-R, R)}}\right] \leqslant \frac{c_{5} e^{-\lambda t_{0}}}{t_{0}\left(h(R)+R^{-1}\left|b_{R}\right|\right)} .
$$

Thus, if we set $t_{0}=c_{6} /\left(h(R)+R^{-1} b_{R}\right)$ and $\lambda=c_{7}\left(h(R)+R^{-1} b_{R}\right)$, where $c_{6}$ and $c_{7}$ are such that $c_{6} \leqslant c_{4} /\left(4 c_{5}\left(c_{\alpha}+e^{-1}\right)\right)$ and $c_{7} \geqslant c_{6}^{-1} \ln \frac{4 c_{5}\left(c_{\alpha}+e^{-1}\right)}{c_{4} c_{6}}$, then putting everything together yields

$$
G_{(-R, R)}(x, y) \geqslant \frac{c_{4}}{2} \frac{1}{\lambda h^{-1}(\lambda)} .
$$

Since by Grzywny and Szczypkowski (2020, Lemma 2.10) we have $\lambda \approx h(R)$, using scaling properties of $h^{-1}$ we get that

$$
G_{(-R, R)}(x, y) \gtrsim \frac{1}{R h(R)}, \quad|x|,|y| \leqslant \delta_{1} R,
$$

with some $\delta_{1} \in(0,1]$, and the claim follows by Proposition 2.2.

Proposition 5.2. Suppose $\operatorname{Re} \psi \in \operatorname{WLSC}(\alpha, \chi)$ for some $\alpha>1$ and $\chi \in(0,1]$. There is $\delta_{2} \leqslant \delta_{1}$ dependent only on the scalings such that for any $R>0$ any non-empty $A \subset\left(-\delta_{2} R, \delta_{2} R\right)$,

$$
\mathbb{P}^{x}\left(T_{A}<\tau_{(-R, R)}\right) \geqslant \frac{1}{2}, \quad|x| \leqslant \delta_{2} R .
$$

Proof: Let $|a| \leqslant R / 4$ and $D=(-R / 2,0) \cup(0, R / 2)$. By Grzywny (2014, Lemma 3$)$ and Proposition 2.4, there is $C_{1}>0$ such that for $|x-a| \leqslant R / 4$,

$$
\begin{aligned}
\mathbb{P}^{x}\left(T_{a}>\tau_{(-R, R)}\right) & \leqslant \mathbb{P}^{x-a}\left(T_{0}>\tau_{(-R / 2, R / 2)}\right) \\
& \leqslant C_{1} h(R / 2) \mathbb{E}^{x-a} \tau_{D} \leqslant 8 C_{1} R h(R) H(x-a) .
\end{aligned}
$$

In view of Proposition 2.2, there is $C_{2}>0$ dependent only on the scalings such that

$$
\mathbb{P}^{x}\left(T_{a}>\tau_{(-R, R)}\right) \leqslant C_{2} \frac{H(x-a)}{H(R)}, \quad|x-a|<R / 4 .
$$

Since by Proposition 2.2, $H \in \operatorname{WLSC}(\alpha-1, \tilde{\chi})$ for some $\tilde{\chi} \in(0,1]$, we can pick $\delta_{2}<1 / 2$ such that

$$
\mathbb{P}^{x}\left(T_{a}>\tau_{(-R, R)}\right) \leqslant \frac{1}{2}, \quad|x-a|<2 \delta_{2} R .
$$

It follows that if $x \in A \subset\left(-\delta_{2} R, \delta_{2} R\right)$ and $a \in A$, then

$$
\mathbb{P}^{x}\left(T_{A} \geqslant \tau_{(-R, R)}\right) \leqslant \mathbb{P}^{x}\left(T_{a}>\tau_{(-R, R)}\right) \leqslant \frac{1}{2},
$$

and the proof is completed.

Denote $R_{0}=\delta_{2} R$, where $\delta_{2}$ is taken from Proposition 5.2.

Proposition 5.3. Suppose $\operatorname{Re} \psi \in \operatorname{WLSC}(\alpha, \chi)$ for some $\alpha>1$ and $\chi \in(0,1]$. Then for any $R>0$ and any non-negative function $F$ such that $(\operatorname{supp} F)^{c} \subset$ $(-R, R)$,

$$
\mathbb{E}^{x} F\left(X_{\tau_{\left(-R_{0}, R_{0}\right)}}\right) \lesssim \frac{1}{c} \mathbb{E}^{y} F\left(X_{\tau_{(-R, R)}}\right), \quad|x|,|y| \leqslant R_{0},
$$

where $c$ is taken from Lemma 5.1. The implied comparability depends only on the scalings. 
Proof: Let us denote, for any $w \in \mathbb{R}$ and a Borel set $A, \nu(w, A)=\nu(A-w)$. By the Ikeda-Watanabe formula and Lemma 5.1,

$$
\begin{aligned}
\mathbb{E}^{y} F\left(X_{\tau_{(-R, R)}}\right) & \geqslant \int_{(-R, R)^{c}} \int_{-R_{0}}^{R_{0}} F(z) G_{(-R, R)}(y, w) \nu(w, d z) d w \\
& \geqslant \tilde{c} H(R) \int_{(-R, R)^{c}} \int_{-R_{0}}^{R_{0}} F(z) \nu(w, d z) d w .
\end{aligned}
$$

On the other hand, by the Ikeda-Watanabe formula, Proposition 2.3, subadditivity and almost monotonicity of $H$,

$$
\begin{aligned}
\mathbb{E}^{x} F\left(X_{\left.\tau_{\left(-R_{0}, R_{0}\right)}\right)}\right. & \leqslant \int_{(-R, R)^{c}} \int_{-R_{0}}^{R_{0}} F(z) G_{\{0\}^{c}}\left(x+R_{0}, w+R_{0}\right) \nu(w, d z) d w \\
& \lesssim H\left(R_{0}\right) \int_{(-R, R)^{c}} \int_{-R_{0}}^{R_{0}} F(z) \nu(w, d z) d w .
\end{aligned}
$$

Hence,

$$
\mathbb{E}^{x} F\left(X_{\tau_{\left(-R_{0}, R_{0}\right)}}\right) \leqslant \frac{1}{c} \mathbb{E}^{y} F\left(X_{\tau_{(-R, R)}}\right) .
$$

Theorem 5.4. Suppose $\operatorname{Re} \psi \in \operatorname{WLSC}(\alpha, \chi)$ for some $\alpha>1$ and $\chi \in(0,1]$. Then the global scale invariant Harnack inequality holds, i.e. there is a constant $C_{H}$ dependent only on the scalings such that for any $R>0$ and any non-negative harmonic function on $(-R, R)$ we have

$$
\sup _{x \in(-R / 2, R / 2)} h(x) \leqslant C_{H} \inf _{x \in(-R / 2, R / 2)} h(x) .
$$

Proof: Suppose first that $h$ is bounded. Then using the approach of Bass and Levin (2002) we infer that there exist constants $c_{1}=c_{1}(\alpha, \chi)$ and $a=a(\alpha, \chi) \in(0,1]$ such that for any non-negative, bounded and harmonic function on $(-R, R)$,

$$
\sup _{x \in(-a R, a R)} h(x) \leqslant c_{1} \inf _{x \in(-a R, a R)} h(x) .
$$

For the justification of that claim we observe that Lemma 5.1 is an analogue of Bass and Levin (2002, Lemma 3.2), Proposition 5.2 corresponds to Bass and Levin (2002, Proposition 3.4) and Proposition 5.3 mirrors Bass and Levin (2002, Proposition 3.5). We also observe that Bass and Levin (2002, Lemma 3.3) in our setting follows immediately from Pruitt's estimates (Pruitt, 1981) and Proposition 2.2. With these tools at our disposal, one can follow the proof of Bass and Levin (2002, Theorem 3.6) almost directly.

Now we apply a standard chain argument to get

$$
\sup _{x \in(-R / 2, R / 2)} h(x) \leqslant C_{H} \inf _{x \in(-R / 2, R / 2)} h(x) .
$$

Indeed, observe that $h$ is harmonic in $(a R-(1-a) R, a R+(1-a) R)$. Thus, after applying (5.2) to the function $\tilde{h}(x)=h(x-a R)$ we conclude that (5.2) holds true also for $x \in(-(a+a(1-a)) R,(a+a(1-a)) R)$ with the constant $c_{1}^{2}$. By verbatim repetition of that argument we get that (5.2) holds true for $x \in\left(-a R \sum_{k=0}^{n-1}(1-\right.$ $\left.a)^{k}, a R \sum_{k=0}^{n-1}(1-a)^{k}\right)=\left(-R\left(1-(1-a)^{n}\right), R\left(1-(1-a)^{n}\right)\right)$ with a constant $c_{1}^{n}$. 
It is clear now that we get the claim with $C_{H}=c_{1}^{n}$ for sufficiently large $n$ which depends only on $a$. It remains to observe that the boundedness assumption on $h$ may be removed in the similar way as in the proof of Song and Vondraček (2004, Theorem 2.4).

Thanks to the Harnack property we are able to prove a relation between renewal functions and their derivatives, and provide a sharp estimate for the Green function of the positive half-line.

Corollary 5.5. Suppose that $\mathbb{E} X_{1}=0$ and $\operatorname{Re} \psi \in \operatorname{WLSC}(\alpha, \chi)$ for some $\alpha>1$ and $\chi \in(0,1]$. Then there is $c \geqslant 1$ such that for all $x>0$,

$$
c^{-1} \frac{V(x)}{x} \leqslant V^{\prime}(x) \leqslant c \frac{V(x)}{x},
$$

and

$$
c^{-1} \frac{\widehat{V}(x)}{x} \leqslant \widehat{V}^{\prime}(x) \leqslant c \frac{\widehat{V}(x)}{x},
$$

In particular, $V^{\prime}, \widehat{V}^{\prime} \in \operatorname{WLSC}(\alpha-2, \tilde{\gamma})$ for some $\tilde{\gamma} \in(0,1]$.

Proof: First, let us consider the second part of the claim. Let $x>0$. Recall that $\widehat{V}^{\prime}$ is harmonic on $(0, \infty)$. Thus, by Theorem 5.4 ,

$$
\widehat{V}(x) \geqslant \int_{x / 2}^{x} \widehat{V}^{\prime}(s) d s \geqslant \frac{1}{2 C_{H}} x \widehat{V}^{\prime}(x) .
$$

On the other hand, since $\operatorname{Re} \psi$ is the same for $\mathbf{X}$ and $\widehat{\mathbf{X}}$, we may apply Grzywny (2019, Lemma 8) for $\widehat{V}$. Let $c_{1}$ be taken from Grzywny (2019, Lemma 8) and $\delta \in\left(0,\left(c_{1} / 2\right)^{1 /(\alpha-1)}\right]$. Then, again by Theorem 5.4 ,

$$
C_{H}(1-\delta) x \widehat{V}^{\prime}(x) \geqslant \int_{\delta x}^{x} \widehat{V}^{\prime}(s) d s=\widehat{V}(x)-\widehat{V}(\delta x) \geqslant\left(1-c_{1}^{-1} \delta^{\alpha-1}\right) \widehat{V}(x) \geqslant \frac{1}{2} \widehat{V}(x) .
$$

Now, the lower scaling property follows immediately by Grzywny (2019, Lemma 8). For the proof of the first part it remains to observe that by the previous remark on the real part of the characteristic exponent, $V$ also satisfies the Harnack inequality (with the same constant) and one can repeat the reasoning above to finish the proof.

Corollary 5.6. Suppose that $\mathbb{E} X_{1}=0$ and $\operatorname{Re} \psi \in \operatorname{WLSC}(\alpha, \chi)$ for some $\alpha>1$ and $\chi \in(0,1]$. Then

$$
G_{(0, \infty)}(x, y) \approx \begin{cases}\widehat{V}(x) V^{\prime}(y), & 0<x \leqslant y, \\ \widehat{V}^{\prime}(x) V(y), & 0<y<x .\end{cases}
$$

The comparability constant depends only on the scaling characteristics.

Proof: First assume that $0<x \leqslant y$. Recall that

$$
G_{(0, \infty)}(x, y)=\int_{0}^{x} \widehat{V}^{\prime}(u) V^{\prime}(y-x+u) d u, \quad 0<x \leqslant y .
$$

Since $V$ is monotone and subadditive, for any $\lambda \geqslant 1$ and $x>0$ we have

$$
V(\lambda x) \leqslant 2 \lambda V(x) .
$$


That, in view of Corollary 5.5, implies that $V^{\prime}$ is almost decreasing, and consequently,

$$
G_{(0, \infty)}(x, y) \gtrsim \int_{0}^{x} \widehat{V}^{\prime}(u) V^{\prime}(y) d u=\widehat{V}(x) V^{\prime}(y) .
$$

Next, let $x<y<2 x$. By Corollary 5.5, Grzywny (2019, Corollary 5), and almost monotonicity of $V^{\prime}$,

$$
G_{(0, \infty)}(x, y) \lesssim \int_{0}^{x} \widehat{V}^{\prime}(u) V^{\prime}(u) d u \approx \int_{0}^{x} \frac{d u}{u^{2} h(u)} .
$$

Using scaling property of $h$, Grzywny (2019, Corollary 5) and Corollary 5.5, we conclude that

$$
G_{(0, \infty)}(x, y) \lesssim \frac{1}{x h(x)} \approx \frac{\widehat{V}(x) V(x)}{x} \lesssim \frac{\widehat{V}(x) V(y)}{y} \lesssim \widehat{V}(x) V^{\prime}(y) .
$$

where the third inequality follows from monotonicity of $V$. Finally, for $y \geqslant 2 x$ we use scaling property of $V^{\prime}$ with index $\alpha-2$ (Corollary 5.5) to obtain

$$
V^{\prime}(y-x+u) \lesssim V^{\prime}(y)\left(\frac{y}{y-x+u}\right)^{2-\alpha} \leqslant 2^{2-\alpha} V^{\prime}(y),
$$

and the first part follows.

If $0<y \leqslant x$ we use the Green function for the dual process to get the claim.

Assume that $\mathbb{E} X_{1}=0$ and $\operatorname{Re} \psi \in \operatorname{WLSC}(\alpha, \chi)$ for some $\alpha>1$ and $\chi \in(0,1]$. By Silverstein (1980, Theorem 1), $V^{\prime}$ is coharmonic on $(0, \infty)$, that is harmonic on $(0, \infty)$ for the dual process $\widehat{\mathbf{X}}$. Since $\operatorname{Re} \psi$ is symmetric, the Harnack inequality for $\widehat{\mathbf{X}}$ holds as well. Thus, by Theorem 5.4 , for any $0<\delta \leqslant w \leqslant u \leqslant w+2 \delta$,

$$
V^{\prime}(u) \leqslant C_{H} V^{\prime}(w)
$$

With that property at hand, proofs of the remaining lemmas in this section follow directly results obtained in Grzywny and Ryznar (2017, Subsection 4.2) and therefore they are omitted.

Lemma 5.7. Suppose that $\mathbb{E} X_{1}=0$ and $\operatorname{Re} \psi \in \operatorname{WLSC}(\alpha, \chi)$ for some $\alpha>1$ and $\chi \in(0,1]$. Let $F(z)$ be non-negative, $F(x) \leqslant F_{1}(x)$ on $\mathbb{R}$ and $F(x+y) \leqslant F_{1}(x)+$ $F_{1}(y)$, for $x, y \in \mathbb{R}$. Suppose that $\mathbb{E}^{x} F\left(X_{\tau_{(0, \infty)}}\right) \leqslant F(x)$ and $\mathbb{E}^{x} F_{1}\left(X_{\tau_{(0, \infty)}}\right) \leqslant F_{1}(x)$ for $x>0$. Then there is $c>0$ such that for any $0<x<1$,

$$
\mathbb{E}^{x}\left[X_{\tau_{(0, \infty)}} \leqslant-2 ; F\left(X_{\tau_{(0, \infty)}}\right)\right] \leqslant c C_{H}^{2} F_{1}^{*}(1) \frac{\widehat{V}(x)}{\widehat{V}(1)} .
$$

The constant $c$ depends only on the scalings.

Proof: Follows directly by proof of Grzywny and Ryznar (2017, Lemma 4.7) with applications of Lemma 4.6 and Lemma 2.9 replaced by Corollary 5.6 and Grzywny (2019, Corollary 5), respectively, and using a function $F_{1}$ instead of subadditivity of $F$.

Lemma 5.8. Suppose $\mathbb{E} X_{1}=0$ and $\operatorname{Re} \psi \in \operatorname{WLSC}(\alpha, \chi)$ for some $\alpha>1$ and $\chi \in(0,1]$. Let $F$ be a non-negative harmonic function on $(0,2 R)$ for some $R>0$. 
Suppose that $r>0$ is such that $\widehat{V}(R) \geqslant 2 \widehat{V}(r) / \tilde{c}$, where $c$ is taken from Proposition 2.5. Then for $0<x<r$,

$$
\frac{F(x)}{F(r)} \geqslant \frac{c}{4} C_{H}^{-1-R / r} \frac{\widehat{V}(x)}{\widehat{V}(r)},
$$

where $C_{H}$ is the constant from the Harnack inequality (5.1).

Proof: Follows directly the proof of Grzywny and Ryznar (2017, Lemma 4.8) with applications of Theorem 4.5 and Lemma 2.11 replaced by Theorem 5.4 and Proposition 2.5 , respectively.

\section{Estimates}

In this Section we prove sharp two-sided estimates on the tail of the first hitting time of the interval. Our main result here is Theorem 6.10. We also provide an analogous estimate for the specific case of spectrally negative Lévy processes. Afterwards, in Subsection 6.1 we point out a large class of non-symmetric Lévy processes which satisfy its assumptions.

We begin with the following estimate on $u^{\lambda}$.

Lemma 6.1. Assume that there exist constants $a>0, b \geqslant 0$ such that $|\operatorname{Im} \psi(\xi)| \leqslant$ $b \operatorname{Re} \psi(\xi), \xi \in \mathbb{R}$ and $a \psi^{*}(x) \leqslant \operatorname{Re} \psi(x), x \geqslant 0$. Then we have

$$
\frac{a}{4\left(1+b^{2}\right)} H\left(\frac{1}{(\operatorname{Re} \psi)^{-1}(\lambda)}\right) \leqslant u^{\lambda}(0) \leqslant \frac{3 \pi^{2}\left(1+b^{2}\right)}{2 a} H\left(\frac{1}{(\operatorname{Re} \psi)^{-1}(\lambda)}\right) .
$$

Proof: Since $|\operatorname{Im} \psi(\xi)| \leqslant b \operatorname{Re} \psi(\xi)$ we have

$$
\frac{1}{\pi\left(1+b^{2}\right)} \int_{0}^{\infty} \frac{d \xi}{\lambda+\operatorname{Re} \psi(\xi)} \leqslant u^{\lambda}(0) \leqslant \frac{1}{\pi} \int_{0}^{\infty} \frac{d \xi}{\lambda+\operatorname{Re} \psi(\xi)} .
$$

Hence, by Grzywny and Ryznar (2017, Lemma 2.15), for $\lambda>0$,

$$
u^{\lambda}(0) \geqslant \frac{a}{4 \pi\left(1+b^{2}\right)} \int_{0}^{\infty}\left(1-\cos \left(s /(\operatorname{Re} \psi)^{-1}(\lambda)\right)\right) \frac{d s}{\operatorname{Re} \psi(s)}
$$

and

$$
u^{\lambda}(0) \leqslant \frac{3 \pi^{2}\left(1+b^{2}\right)}{2 a} \int_{0}^{\infty}\left(1-\cos \left(s /(\operatorname{Re} \psi)^{-1}(\lambda)\right)\right) \frac{d s}{\operatorname{Re} \psi(s)} .
$$

Using $|\operatorname{Im} \psi(\xi)| \leqslant b \operatorname{Re} \psi(\xi)$ we infer that

$$
\pi H(x) \leqslant \int_{0}^{\infty}(1-\cos (x s)) \frac{d s}{\operatorname{Re} \psi(s)} \leqslant\left(1+b^{2}\right) \pi H(x),
$$

which ends the proof.

Lemma 6.2. Suppose that $\mathbb{E} X_{1}=0$ and $\operatorname{Re} \psi \in \operatorname{WLSC}(\alpha, \chi)$ for some $\alpha>1$ and $\chi \in(0,1]$. Then there exists $c=c(\alpha, \chi)$ such that, for any $a, x>0$,

$$
K^{\lambda}(x) \geqslant c\left(1-e^{-a}\right) u^{\lambda}(0), \quad \lambda \geqslant a h(x) .
$$

Proof: Since

$$
K^{\lambda}(x)=\lambda u^{\lambda}(0) \mathcal{L}\left[\mathbb{P}^{x}\left(T_{0}>\cdot\right)\right](\lambda),
$$


it is enough to prove that $\mathcal{L}\left[\mathbb{P}^{x}\left(T_{0}>\cdot\right)\right](\lambda) \geqslant c / \lambda$, if $\lambda \geqslant a h(x)$. Using estimates of the tail distribution of the first exit time from the positive half-line (Grzywny, 2019, Theorem 6) we conclude that there is $c_{1}$ such that

$$
\begin{aligned}
\mathcal{L}\left[\mathbb{P}^{x}\left(T_{0}>\cdot\right)\right](\lambda) & \geqslant c_{1} \int_{0}^{\infty}\left(1 \wedge \frac{\hat{V}(x)}{\hat{V}\left(h^{-1}(1 / s)\right)}\right) e^{-\lambda s} d s \\
& \geqslant c_{1} \int_{0}^{1 / h(x)} e^{-\lambda s} d s \geqslant c_{1}\left(1-e^{-a}\right) \lambda^{-1} .
\end{aligned}
$$

Proposition 6.3. Assume that there exist constants $a>0, b \geqslant 0$ such that $|\operatorname{Im} \psi(\xi)| \leqslant b \operatorname{Re} \psi(\xi), \xi \in \mathbb{R}$ and $a \psi^{*}(x) \leqslant \operatorname{Re} \psi(x)$ for $x \geqslant 0$. Then

$$
\mathbb{P}^{x}\left(T_{0}>t\right) \leqslant \frac{4(e-1)}{e} \frac{\left(1+b^{2}\right)}{a} \frac{H(x)}{H\left(1 /(\operatorname{Re} \psi)^{-1}(1 / t)\right)} \wedge 1 .
$$

Proof: Recall that

$$
\lambda \mathcal{L}\left(\mathbb{P}^{x}\left(T_{0}>\cdot\right)\right)(\lambda)=\left[1-\mathbb{E}^{x} e^{-\lambda T_{0}}\right]=\frac{u^{\lambda}(0)-u^{\lambda}(-x)}{u^{\lambda}(0)}=\frac{K^{\lambda}(-x)}{u^{\lambda}(0)} .
$$

By Lemma 6.1,

$$
\mathcal{L}\left(\mathbb{P}^{x}\left(T_{0}>\cdot\right)\right)(\lambda) \leqslant \frac{4\left(1+b^{2}\right)}{a} \frac{H(x)}{H\left(1 /(\operatorname{Re} \psi)^{-1}(\lambda)\right)} .
$$

Therefore, using Bogdan et al. (2014, Lemma 5) we conclude that

$$
\mathbb{P}^{x}\left(T_{0}>t\right) \leqslant \frac{e}{e-1} \frac{4\left(1+b^{2}\right)}{a} \frac{H(x)}{H\left(1 /(\operatorname{Re} \psi)^{-1}(1 / t)\right)} .
$$

Corollary 6.4. Assume that $\mathbb{E} X_{1}=0$ and $\operatorname{Re} \psi \in \operatorname{WLSC}(\alpha, \chi)$ for some $\alpha>1$ and $\chi \in(0,1]$. Then there is $c>0$ such that for all $t>0$,

$$
\mathbb{P}^{x}\left(T_{0}>t\right) \leqslant c \frac{H(x)}{H\left(h^{-1}(1 / t)\right)} \wedge 1 .
$$

The constant $c$ depends only on the scalings.

Proof: Using Grzywny (2019, Lemma 12) and Grzywny and Szczypkowski (2020, Remark 3.2) we see that the assumptions of Proposition 6.3 are satisfied. Now it remains to apply comparability of $1 /(\operatorname{Re} \psi)^{-1}$ and $h^{-1}$ together with Proposition 2.2.

Lemma 6.5. Suppose $\mathbb{E} X_{1}=0$ and $\operatorname{Re} \psi \in \operatorname{WLSC}(\alpha, \chi)$ for some $\alpha>1$ and $\chi \in(0,1]$. If $x>1$ and $t<1 / h(1)$ then

$$
\mathbb{P}^{x}\left(T_{B_{1}}>t\right) \approx \frac{\hat{V}(x-1)}{\hat{V}\left(h^{-1}(1 / t)\right)} \wedge 1 .
$$

The comparability constant depends only on the scalings. 
Proof: Of course, the lower bound is a consequence of the estimates on the tail for the first exit time from a half-line, that is Grzywny (2019, Theorem 6). By subadditivity of $\hat{V}$, it is enough to consider $1<x<1+h^{-1}(1 / t) / 2$, because if $x$ is larger, by the lower bound the probability is comparable to 1 .

To prove the estimate from the above let us denote $r=h^{-1}(1 / t)$. Notice that $r<1$ and we have

$$
\mathbb{P}^{x}\left(T_{B_{1}}>t\right) \leqslant \mathbb{P}^{x}\left(\tau_{(1,1+r)}>t\right)+\mathbb{P}^{x}\left(\left|X_{\tau_{(1,1+r)}}-1\right|>r\right) .
$$

Combining Grzywny (2014, Lemma 3) and Grzywny (2019, the proof of Proposition 4) we obtain

$$
\mathbb{P}^{x}\left(\left|X_{\tau_{(1,1+r)}}-1\right|>r\right) \leqslant c \mathbb{E}^{x} \tau_{(1,1+r)} h(r) \leqslant c \hat{V}(x-1) V(r) h(r),
$$

for some $c>0$. Finally by Grzywny (2019, Corollary 5),

$$
\mathbb{P}^{x}\left(\left|X_{\tau_{(1,1+r)}}-1\right|>r\right) \leqslant c \frac{\hat{V}(x-1)}{\hat{V}(r)} .
$$

This together with Grzywny (2019, Theorem 6) imply

$$
\mathbb{P}^{x}\left(T_{B_{1}}>t\right) \leqslant c \frac{\hat{V}(x-1)}{\hat{V}(r)} .
$$

Lemma 6.6. Assume that $\mathbb{E} X_{1}=0$ and $\operatorname{Re} \psi \in \operatorname{WLSC}(\alpha, \chi)$ for some $\alpha>1$ and $\chi \in(0,1]$. If $x>1$ and $t \geqslant 1 / h(1)$ then

$$
\mathbb{P}^{x}\left(T_{B_{1}}>t\right) \leqslant c \frac{\hat{V}(x-1)}{\hat{V}(x)} \frac{H(x)}{H\left(h^{-1}(1 / t)\right)} \wedge 1 \approx \frac{\hat{V}(x-1)}{\hat{V}(x)} \frac{H(x)}{t / h^{-1}(1 / t)} \wedge 1 .
$$

The constant $c$ depends only on the scalings.

Proof: If $x \geqslant 2$ we have, by subadditivity and monotonicity of $\hat{V}, \frac{\hat{V}(x-1)}{\hat{V}(x)} \geqslant \frac{1}{2}$, hence the claim follows from Grzywny (2019, Lemma 12) and Corollary 6.4.

Let $1<x<2$. By Grzywny (2019, Theorem 6),

$$
\mathbb{P}^{x}\left(\tau_{(1, \infty)}>t\right) \approx 1 \wedge \frac{\hat{V}(x-1)}{\hat{V}\left(h^{-1}(1 / t)\right)} .
$$

Since $t>1 / h(1)$, using subadditivity of $\hat{V}$ and Grzywny and Szczypkowski (2020, Lemma 2.1) we obtain

$$
\begin{aligned}
\mathbb{P}^{x}\left(\tau_{(1, \infty)}>t / 2\right) & \leqslant c_{1} \frac{\hat{V}(x-1)}{\hat{V}(1)} \frac{\hat{V}(1)}{\hat{V}\left(h^{-1}(1 / t)\right)} \leqslant c_{2} \frac{\hat{V}(x-1)}{\hat{V}(1)} \mathbb{P}^{2}\left(\tau_{(1, \infty)}>t\right) \\
& \leqslant c_{3} \frac{\hat{V}(x-1)}{\hat{V}(x)} \mathbb{P}^{1}\left(T_{0}>t\right) .
\end{aligned}
$$

Since

$$
\mathbb{P}^{x}\left(T_{B_{1}}>t\right) \leqslant \mathbb{P}^{x}\left(\tau_{(1, \infty)}>t / 2\right)+\mathbb{E}^{x} \mathbb{P}^{X_{\tau_{(1, \infty)}}}\left(T_{B_{1}}>t / 2\right),
$$


due to Proposition 2.2 and Corollary 6.4, it is enough to estimate the second term. We have

$$
\begin{aligned}
\mathbb{E}^{x} \mathbb{P}^{X_{\tau(1, \infty)}}\left(T_{B_{1}}>t / 2\right) & \leqslant \mathbb{E}^{x}\left[X_{\tau_{(1, \infty)}} \leqslant-1 ; \mathbb{P}^{X_{\tau_{(1, \infty)}}}\left(T_{1}>t / 2\right)\right] \\
& =\mathbb{E}^{x-1}\left[X_{\tau_{(0, \infty)}} \leqslant-2 ; \mathbb{P}^{X_{\tau_{(0, \infty)}}}\left(T_{0}>t / 2\right)\right] .
\end{aligned}
$$

Let $F(z)=\mathbb{P}^{z}\left(T_{0}>t / 2\right)$. Observe that

$$
\begin{aligned}
F(z) & =\mathbb{P}^{z}\left(\tau_{(0, \infty)}>t / 2\right)+\mathbb{E}^{z}\left[\tau_{(0, \infty)} \leqslant t / 2 ; \mathbb{P}^{X_{\tau_{(0, \infty)}}}\left(T_{0}>t / 2-\tau_{(0, \infty)}\right)\right] \\
& \geqslant \mathbb{E}^{z}\left[\mathbb{P}^{X_{\tau_{(0, \infty)}}}\left(T_{0}>t / 2\right)\right] \\
& =\mathbb{E}^{z} F\left(X_{\tau_{(0, \infty)}}\right) .
\end{aligned}
$$

Furthermore,

$$
\begin{aligned}
F(x+y) & \leqslant \mathbb{P}^{x+y}\left(T_{x}>t / 4\right)+\mathbb{E}^{x}\left[T_{x} \leqslant t / 4 ; \mathbb{P}^{X_{T_{x}}}\left(T_{0}>t / 4\right)\right] \\
& \leqslant \mathbb{P}^{y}\left(T_{0}>t / 4\right)+\mathbb{P}^{x}\left(T_{0}>t / 4\right) .
\end{aligned}
$$

Hence, $F$ and $F_{1}(z)=\mathbb{P}^{z}\left(T_{0}>t / 4\right)$ satisfy the assumptions of Lemma 5.7. Therefore, the conclusion follows from Lemma 5.7 and Proposition 6.3.

Lemma 6.7. Assume $\mathbb{E} X_{1}=0$ and $\operatorname{Re} \psi \in \operatorname{WLSC}(\alpha, \chi)$ for some $\alpha>1$ and $\chi \in(0,1]$. If $x_{0}>1,1<x \leqslant x_{0}$ and $t>1 / h(1)$, then there is $c=c\left(x_{0}, \alpha, \chi\right)>0$ such that

$$
\mathbb{P}^{x}\left(T_{B_{1}}>t\right) \geqslant c \frac{\hat{V}(x-1)}{\hat{V}\left(x_{0}\right)} \mathbb{P}^{x_{0}}\left(T_{0}>2 t\right) .
$$

Proof: With Lemma 5.8 and Grzywny (2019, Theorem 6) at hand, the proof is the same as the first part of the proof of Grzywny and Ryznar (2017, Lemma 5.4) and therefore it is omitted.

Lemma 6.8. Assume that $\mathbb{E} X_{1}=0$ and $\operatorname{Re} \psi \in \operatorname{WLSC}(\alpha, \chi)$ for some $\alpha>1$ and $\chi \in(0,1]$. Suppose that there exist constants $c>0$ and $a>0$ such that for $x>0$, $K^{\lambda}(x) \geqslant c H(x), \lambda \leqslant a h(x)$. Then there is $\tilde{c}=\tilde{c}(a, \alpha, c)>0$ such that

$$
\mathbb{P}^{x}\left(T_{0}>t\right) \geqslant \tilde{c}\left(1 \wedge \frac{H(x)}{H\left(h^{-1}(1 / t)\right)}\right), \quad x, t>0 .
$$

We remark that in case of symmetric Lévy processes the last assumption follows from Grzywny and Ryznar (2017, Lemma 2.15).

Proof: By Lemma 6.1, comparability of $h$ and $\psi$, and Proposition 2.2,

$$
\lambda \mathcal{L}\left(\mathbb{P}^{x}\left(T_{0}>\cdot\right)\right)(\lambda) \approx \frac{K^{\lambda}(x)}{H\left(h^{-1}(\lambda)\right)} .
$$

Let $x>0, \lambda>0$ and $s>1$. Combining Lemma 6.2 with the assumption on $K^{\lambda}$ we obtain, for $\lambda \geqslant a h(x)$ or $\lambda s \leqslant a h(x)$,

$$
\frac{K^{\lambda s}(x)}{K^{\lambda}(x)} \lesssim 1
$$

If $\lambda s \geqslant a h(x) \geqslant \lambda$ we have, by Lemma 6.1 and almost monotonicity of $H$,

$$
\frac{K^{\lambda s}(x)}{K^{\lambda}(x)} \approx \frac{u^{\lambda}(0)}{H(x)} \approx \frac{H\left(h^{-1}(\lambda s)\right)}{H(x)} \lesssim \frac{H\left(h^{-1}(a h(x))\right)}{H(x)} \lesssim 1 .
$$


Thus,

and consequently,

$$
\frac{K^{\lambda s}(x)}{K^{\lambda}(x)} \leqslant c
$$

$$
\frac{\mathcal{L}\left(\mathbb{P}^{x}\left(T_{0}>\cdot\right)\right)(\lambda s)}{\mathcal{L}\left(\mathbb{P}^{x}\left(T_{0}>\cdot\right)\right)(\lambda)} \leqslant c \frac{\lambda}{\lambda s} \frac{K^{\lambda s}(x)}{K^{\lambda}(x)} \frac{H\left(h^{-1}(\lambda)\right)}{H\left(h^{-1}(\lambda s)\right)} \leqslant c \frac{h^{-1}(\lambda s)}{h^{-1}(\lambda)} \leqslant c s^{-1 / 2},
$$

where $c$ depends only on the scalings and $a$. Hence, by Bogdan et al. (2014, Lemma 13) there exists a constant $c_{1}$ that depends only on the scalings such that

$$
\mathbb{P}^{x}\left(T_{0}>t\right) \geqslant c_{1} \frac{K^{1 / t}(x)}{H\left(\frac{1}{\psi^{-1}(1 / t)}\right)} .
$$

For $t>1 / a h(x)$ we get the claim by the comparability $K^{1 / t}$ with $K$ and for $t \leqslant$ $1 /(a h(x))$ we use estimates for the positive half-line (Grzywny, 2019, Theorem 6).

Proposition 6.9. Assume $\mathbb{E} X_{1}=0$ and $\operatorname{Re} \psi \in \operatorname{WLSC}(\alpha, \chi)$ for some $\alpha>1$ and $\chi \in(0,1]$. Suppose that there exist constants $c>0$ and $a>0$ such that for $x>0$, $K^{\lambda}(x) \geqslant c H(x), \lambda \leqslant a h(x)$. Then there is $x_{0} \geqslant 2$, which depends only on the scaling characteristics and $a$, such that for $x \geqslant x_{0}$ we have, for $t>1 / h(1)$,

$$
\mathbb{P}^{x}\left(T_{B_{1}}>t\right) \geqslant \tilde{c}\left(\frac{H(|x|)}{H\left(h^{-1}(1 / t)\right)} \wedge 1\right) \approx\left(\frac{H(|x|)}{t / h^{-1}(1 / t)} \wedge 1\right) .
$$

The constant $\tilde{c}$ depends only on the scalings and a.

The proof is very similar to the proof of Grzywny and Ryznar (2017, Proposition 5.3 ) with modifications like in the proof above therefore it is omitted.

We now proceed to the proof of the main result of this Section.

Theorem 6.10. Suppose that $\mathbb{E} X_{1}=0$ and $\operatorname{Re} \psi \in \operatorname{WLSC}(\alpha, \chi)$ for some $\alpha>1$ and $\chi \in(0,1]$. Then for any $R>0$ and $x>R$,

$$
\mathbb{P}^{x}\left(T_{B_{R}}>t\right) \approx \frac{\widehat{V}(x-R)}{\widehat{V}\left(h^{-1}(1 / t)\right)} \wedge 1, \quad t<1 / h(R) .
$$

Furthermore, if we additionally assume that there exist constants $c_{1}>0$ and $a>0$ such that for $x>0, K^{\lambda}(x) \geqslant c_{1} H(x), \lambda \leqslant a h(x)$, then

$\mathbb{P}^{x}\left(T_{B_{R}}>t\right) \approx \frac{\widehat{V}(x-1)}{\widehat{V}(x)} \frac{H(x)}{H\left(h^{-1}(1 / t)\right)} \wedge 1 \approx \frac{\widehat{V}(x-1)}{\widehat{V}(x)} \frac{H(x)}{t / h^{-1}(1 / t)} \wedge 1, \quad t \geqslant 1 / h(R)$.

Proof: The case $R=1$ follows by Lemmas 6.5 and 6.6, and Proposition 6.9. Now we may proceed as in the proof of Grzywny and Ryznar (2017, Theorem 5.5) to obtain the claim for any $R>0$.

Let us turn our attention to the specific class of Lévy processes.

Lemma 6.11. Suppose $\mathbb{E} X_{1}=0, \operatorname{Re} \psi \in \operatorname{WLSC}(\alpha, \chi)$ for some $\alpha>1, \chi \in(0,1]$, and let $R \in[0, \infty)$. Assume that

$$
\int_{0}^{1} \frac{\nu(y, \infty)}{h(y)} \frac{d y}{y}<\infty
$$


Then

$$
\mathbb{P}^{x}\left(T_{B_{R}}>t\right) \approx \frac{H(x-R)}{H\left(h^{-1}(1 / t)\right)} \wedge 1, \quad R<x<R+1,0<t<1 / h(1) .
$$

Proof: A consequence of Proposition 14 and Corollary 5 in Grzywny (2019) is $\tilde{V}(x) \approx \frac{1}{x h(x)}, 0<x \leqslant 1$. That together with Proposition 2.2 imply $\tilde{V}(x) \approx$ $H(x), 0 \leqslant x \leqslant 1$. Hence the claim holds due to Grzywny (2019, Theorem 6) and Corollary 6.4.

Similarly the consequence of Grzywny (2019, Proposition 15 and Section 3) is the following.

Lemma 6.12. Suppose $\mathbb{E} X_{1}=0$ and $\operatorname{Re} \psi \in \operatorname{WLSC}(\alpha, \chi)$ with $\alpha>1, \chi \in(0,1]$, and let $R \in[0, \infty)$. Assume that any of the following holds true:

(i) $\mathbb{E} X_{1}^{2}<\infty$,

(ii) there are $C, r>0$ such that $\nu(x, \infty) \leqslant C \nu(-\infty,-x), x>r$, and

$$
\int_{1}^{\infty} \frac{\nu(y, \infty)}{h(y)} \frac{d y}{y}<\infty
$$

Then

$$
\mathbb{P}^{x}\left(T_{B_{R}}>t\right) \approx \frac{H(x-R)}{H\left(h^{-1}(1 / t)\right)} \wedge 1, \quad x \geqslant R+1, t>0 .
$$

Combining the above two lemmas and the fact that for spectrally negative processes $T_{0}=\tau_{(-\infty, 0)}$ if the process starts from negative half-line, we obtain the following result.

Corollary 6.13. Suppose $\mathbb{E} X_{1}=0$ and $\operatorname{Re} \psi \in \operatorname{WLSC}(\alpha, \chi)$ with $\alpha>1, \chi \in(0,1]$, and let $R \in[0, \infty)$. Assume that $\mathbf{X}$ is spectrally negative, i.e. $\nu(0, \infty)=0$. Then

$$
\mathbb{P}^{x}\left(T_{B_{R}}>t\right) \approx \frac{H(x-R)}{H\left(h^{-1}(1 / t)\right)} \wedge 1, \quad x>R, t>0,
$$

and

$$
\mathbb{P}^{x}\left(T_{B_{R}}>t\right) \approx \frac{|x+R|}{h^{-1}(1 / t)} \wedge 1, \quad x<-R, t>0 .
$$

6.1. A class of processes which satisfy the assumptions of Theorem 6.10. Let us now provide an example of a class of non-symmetric Lévy processes which satisfy the assumptions of Theorem 6.10. As one can suspect, the main difficulty here is the lower estimate on $K^{\lambda}$ for small $\lambda$, which is far from obvious for general nonsymmetric process, even if the remaining two assumptions are satisfied. Note that if process is symmetric then the third assumption follows from Grzywny and Ryznar (2017, Lemma 2.15).

Let $\nu$ be of the form (3.2) such that $\operatorname{Re} \psi \in \operatorname{WLSC}(\alpha, \chi)$, for some $\alpha>1$ and $\chi \in(0,1]$. Since $\int_{|z| \geqslant 1}|z| \nu(d z)<\infty$, the characteristic exponent $\psi$ is differentiable and

$$
(\operatorname{Im} \psi)^{\prime}(\xi)=\int_{\mathbb{R}} z(1-\cos \xi z) \nu(d z)=\left(C_{u}-C_{d}\right) \int_{0}^{\infty} z(1-\cos \xi z) \nu_{0}(d z), \quad \xi \in \mathbb{R} .
$$

Now we specify $\nu_{0}$. Assume that $0<\beta_{1} \leqslant \beta_{2}<1$ and $0<a_{2} \leqslant 1 \leqslant a_{1}$. Let $\nu_{0}(d z)=\frac{f(z)}{z^{2}} d z$, where $f$ is non-negative, non-increasing and satisfies

$$
a_{2} \lambda^{-\beta_{2}} f(z) \leqslant f(\lambda z) \leqslant a_{1} \lambda^{-\beta_{1}} f(z), \quad \lambda>1, z>0 .
$$


For such $\nu_{0}$ it is easy to verify that $\operatorname{Re} \psi$ is non-decreasing on $[0, \infty)$ and by Bogdan et al. (2014, Proposition 28), there is $c_{1}=c_{1}\left(\beta_{1}, \beta_{2}, a_{1}, a_{2}\right)$ such that

$$
\left|(\operatorname{Im} \psi)^{\prime}(\xi)\right| \leqslant c\left|C_{u}-C_{d}\right| f(1 / \xi) \leqslant c_{1} \frac{\left|C_{u}-C_{d}\right|}{C_{u}+C_{d}} \frac{\operatorname{Re} \psi(\xi)}{\xi}, \quad \xi \in \mathbb{R} .
$$

Next, we obtain the lower bound for $K^{\lambda}$ for small $\lambda$. We have, for $x>0$,

$$
\begin{aligned}
K^{\lambda}(x) & =\frac{1}{\pi} \int_{0}^{\infty}(1-\cos x \xi) \frac{\lambda+\operatorname{Re} \psi(\xi)}{(\lambda+\operatorname{Re} \psi(\xi))^{2}+(\operatorname{Im} \psi(\xi))^{2}} d \xi \\
& +\frac{1}{\pi} \int_{0}^{\infty} \sin x \xi \frac{\operatorname{Im} \psi(\xi)}{(\lambda+\operatorname{Re} \psi(\xi))^{2}+(\operatorname{Im} \psi(\xi))^{2}} d \xi \\
& =\frac{1}{2} H^{\lambda}(x)+\mathrm{I}_{\lambda}(x) .
\end{aligned}
$$

Since $|\operatorname{Im} \psi(\xi)| \leqslant c_{2} \operatorname{Re} \psi(\xi), \xi \in \mathbb{R}$, where $c_{2}=c_{2}\left(\beta_{1}, a_{1}\right)$, by Grzywny and Ryznar (2017, Lemma 2.15), for $x>0$ and $\lambda \leqslant h(x)$,

$$
H^{\lambda}(x) \geqslant \frac{1}{\pi\left(1+c_{2}^{2}\right)} \int_{0}^{\infty}(1-\cos x \xi) \frac{d \xi}{\lambda+\operatorname{Re} \psi(\xi)} \geqslant c_{3} \frac{1}{x h(x)},
$$

where $c_{3}$ depends only on $\beta_{1}$ and $a_{1}$. The integration by parts implies, for $x>0$,

$$
\pi x \mathrm{I}_{\lambda}(x)=\int_{0}^{\infty}(1-\cos x \xi) \frac{g(\xi)}{\left((\lambda+\operatorname{Re} \psi(\xi))^{2}+\left(\operatorname{Im} \psi(\xi)^{2}\right)^{2}\right.} d \xi,
$$

where

$$
\begin{aligned}
g(\xi) & =2 \operatorname{Im} \psi(\xi)\left((\operatorname{Re} \psi)^{\prime}(\xi)(\lambda+\operatorname{Re} \psi(\xi))+(\operatorname{Im} \psi)^{\prime}(\xi) \operatorname{Im} \psi(\xi)\right) \\
& -(\operatorname{Im} \psi)^{\prime}(\xi)\left((\lambda+\operatorname{Re} \psi(\xi))^{2}+\left(\operatorname{Im} \psi(\xi)^{2}\right) .\right.
\end{aligned}
$$

Assume that $C_{u} \geqslant C_{d}$, then $\operatorname{Im} \psi,(\operatorname{Im} \psi)^{\prime},(\operatorname{Re} \psi)^{\prime}$ are non-negative on the positive half-line, therefore

$$
\begin{aligned}
\pi x \mathrm{I}_{\lambda}(x) & \geqslant-\int_{0}^{\infty}(1-\cos x \xi) \frac{(\operatorname{Im} \psi)^{\prime}(\xi)}{(\lambda+\operatorname{Re} \psi(\xi))^{2}+\left(\operatorname{Im} \psi(\xi)^{2}\right.} d \xi \\
& \geqslant-c_{1} \frac{C_{u}-C_{d}}{C_{u}+C_{d}} \int_{0}^{\infty}(1-\cos x \xi) \frac{\operatorname{Re} \psi(\xi)}{\xi\left((\lambda+\operatorname{Re} \psi(\xi))^{2}+\left(\operatorname{Im} \psi(\xi)^{2}\right)\right.} d \xi \\
& \geqslant-c_{1} \frac{C_{u}-C_{d}}{C_{u}+C_{d}} \int_{0}^{\infty}(1-\cos x \xi) \frac{1}{\xi \operatorname{Re} \psi(\xi)} d \xi \\
& \geqslant-c_{4} \frac{C_{u}-C_{d}}{C_{u}+C_{d}} \frac{1}{\operatorname{Re} \psi(1 / x)},
\end{aligned}
$$

where in the last inequality we used Bogdan et al. (2014, Corollary 22) and $c_{4}$ depends only on $\beta_{1}, \beta_{2}, a_{1}$ and $a_{2}$. Finally we obtain

$$
K^{\lambda}(x) \geqslant \frac{1}{x h(x)}\left(c_{3}-\frac{c_{4}}{\pi} \frac{C_{u}-C_{d}}{C_{u}+C_{d}}\right), \quad \lambda \leqslant h(x) .
$$

Hence, for small $\frac{C_{u}-C_{d}}{C_{u}+C_{d}}$ we have, for $x>0$,

$$
K^{\lambda}(x) \approx \frac{1}{x h(x)}, \quad \lambda \leqslant h(x) .
$$

For $x<0$ additional assumption on $f(s)-s f^{\prime}(s)$ are needed in order to provide similar calculations. 


\section{References}

Bass, R. F. and Levin, D. A. Harnack inequalities for jump processes. Potential Anal., 17 (4), 375-388 (2002). MR1918242.

Bertoin, J. Lévy processes, volume 121 of Cambridge Tracts in Mathematics. Cambridge University Press, Cambridge (1996). ISBN 0-521-56243-0. MR1406564.

Bingham, N. H., Goldie, C. M., and Teugels, J. L. Regular variation, volume 27 of Encyclopedia of Mathematics and its Applications. Cambridge University Press, Cambridge (1989). ISBN 0-521-37943-1. MR1015093.

Bogdan, K., Grzywny, T., and Ryznar, M. Density and tails of unimodal convolution semigroups. J. Funct. Anal., 266 (6), 3543-3571 (2014). MR3165234.

Bogdan, K., Kumagai, T., and Kwaśnicki, M. Boundary Harnack inequality for Markov processes with jumps. Trans. Amer. Math. Soc., 367 (1), 477-517 (2015). MR3271268.

Bretagnolle, J. Résultats de Kesten sur les processus à accroissements indépendants. In Séminaire de Probabilités, V (Univ. Strasbourg, année universitaire 1969-1970), pp. 21-36. Lecture Notes in Math., Vol. 191 (1971). MR0368175.

Chaumont, L. and Małecki, J. On the asymptotic behavior of the density of the supremum of Lévy processes. Ann. Inst. Henri Poincaré Probab. Stat., 52 (3), 1178-1195 (2016). MR3531705.

Grzywny, T. On Harnack inequality and Hölder regularity for isotropic unimodal Lévy processes. Potential Anal., 41 (1), 1-29 (2014). MR3225805.

Grzywny, T. First exit times from a bounded interval for Lévy processes. ArXiv Mathematics e-prints (2019). arXiv: 1911.05022.

Grzywny, T., Leżaj, Ł., and Trojan, B. Transition densities of subordinators of positive order. ArXiv Mathematics e-prints (2018). arXiv: 1812.06793.

Grzywny, T. and Ryznar, M. Potential theory of one-dimensional geometric stable processes. Colloq. Math., 129 (1), 7-40 (2012). MR3007664.

Grzywny, T. and Ryznar, M. Hitting times of points and intervals for symmetric Lévy processes. Potential Anal., 46 (4), 739-777 (2017). MR3636597.

Grzywny, T. and Szczypkowski, K. Lévy processes: concentration function and heat kernel bounds. Bernoulli, 26 (4), 3191-3223 (2020). MR4140542.

Ikeda, N. and Watanabe, S. On some relations between the harmonic measure and the Lévy measure for a certain class of Markov processes. J. Math. Kyoto Univ., 2, 79-95 (1962). MR142153.

Juszczyszyn, T. and Kwaśnicki, M. Hitting times of points for symmetric Lévy processes with completely monotone jumps. Electron. J. Probab., 20, no. 48, 24 (2015). MR3339868.

Kesten, H. A convolution equation and hitting probabilities of single points for processes with stationary independent increments. Bull. Amer. Math. Soc., 75, 573-578 (1969). MR251797.

Kuznetsov, A., Kyprianou, A. E., Pardo, J. C., and Watson, A. R. The hitting time of zero for a stable process. Electron. J. Probab., 19, no. 30, 26 (2014). MR3183574.

Kwaśnicki, M. Spectral theory for symmetric one-dimensional Lévy processes killed upon hitting the origin. Electron. J. Probab., 17, no. 83, 29 (2012). MR2988398.

Mucha, J. Spectral theory for one-dimensional (non-symmetric) stable processes killed upon hitting the origin. ArXiv Mathematics e-prints (2019). arXiv: 1910.12821. 
Oberhettinger, F. Tables of Mellin transforms. Springer-Verlag, New YorkHeidelberg (1974). MR0352890.

Pantí, H. On Lévy processes conditioned to avoid zero. ALEA Lat. Am. J. Probab. Math. Stat., 14 (2), 657-690 (2017). MR3689384.

Park, H., Xiao, Y., and Yang, X. Uniform dimension results for the inverse images of symmetric Lévy processes. J. Theoret. Probab., 33 (4), 2213-2232 (2020). MR4166197.

Peskir, G. The law of the hitting times to points by a stable Lévy process with no negative jumps. Electron. Commun. Probab., 13, 653-659 (2008). MR2466193.

Pitman, E. J. G. On the behavior of the characteristic function of a probability distribution in the neighborhood of the origin. J. Austral. Math. Soc., 8, 423-443 (1968). MR0231423.

Port, S. C. Hitting times and potentials for recurrent stable processes. J. Analyse Math., 20, 371-395 (1967). MR217877.

Pruitt, W. E. The growth of random walks and Lévy processes. Ann. Probab., 9 (6), 948-956 (1981). MR632968.

Rogozin, B. A. The local behavior of processes with independent increments. Teor. Verojatnost. i Primenen., 13, 507-512 (1968). MR0242261.

Sato, K. Lévy processes and infinitely divisible distributions, volume 68 of Cambridge Studies in Advanced Mathematics. Cambridge University Press, Cambridge (1999). ISBN 0-521-55302-4. MR1739520.

Silverstein, M. L. Classification of coharmonic and coinvariant functions for a Lévy process. Ann. Probab., 8 (3), 539-575 (1980). MR573292.

Simon, T. Hitting densities for spectrally positive stable processes. Stochastics, 83 (2), 203-214 (2011). MR2800088.

Song, R. and Vondraček, Z. Harnack inequality for some classes of Markov processes. Math. Z., 246 (1-2), 177-202 (2004). MR2031452.

Yano, K. On harmonic function for the killed process upon hitting zero of asymmetric Lévy processes. J. Math-for-Ind., 5A, 17-24 (2013). MR3072331.

Yano, K., Yano, Y., and Yor, M. On the laws of first hitting times of points for onedimensional symmetric stable Lévy processes. In Séminaire de Probabilités XLII, volume 1979 of Lecture Notes in Math., pp. 187-227. Springer, Berlin (2009). MR2599211. 